Portland State University

PDXScholar

Summer 8-23-2013

\title{
Identification of Markov Processes within a Wind Turbine Array Boundary Layer
}

Matthew Scott Melius

Portland State University

Follow this and additional works at: https://pdxscholar.library.pdx.edu/open_access_etds

Part of the Mechanical Engineering Commons, and the Power and Energy Commons Let us know how access to this document benefits you.

\section{Recommended Citation}

Melius, Matthew Scott, "Identification of Markov Processes within a Wind Turbine Array Boundary Layer" (2013). Dissertations and Theses. Paper 1422.

https://doi.org/10.15760/etd.1421

This Thesis is brought to you for free and open access. It has been accepted for inclusion in Dissertations and Theses by an authorized administrator of PDXScholar. Please contact us if we can make this document more accessible: pdxscholar@pdx.edu. 
Identification of Markov Processes within a Wind Turbine Array Boundary Layer

by

Matthew Scott Melius

A thesis submitted in partial fulfillment of the requirements for the degree of

\author{
Master of Science \\ in \\ Mechanical Engineering
}

Thesis Committee:

Raúl Bayoán Cal, Chair

Gerald Recktenwald

Edward Zaron

Portland State University

2013 


\begin{abstract}
The Markovianity within a wind turbine array boundary layer is explored for data taken in a wind tunnel containing a model wind turbine array. A stochastic analysis of the data is carried out using Markov chain theory. The data were obtained via hot-wire anemometry thus providing point velocity statistics. The theory of Markovian processes is applied to obtain a statistical description of longitudinal velocity increments inside the turbine wake using conditional probability density functions. It is found that two and three point conditional probability density functions are similar for scale differences larger than the Taylor micro-scale. This result is quantified by use of the Wilcoxon rank-sum test which verifies that this relationship holds independent of initial scale selection outside of the near-wake region behind a wind turbine. Furthermore, at the locations which demonstrate Markovian properties there is a well defined inertial sub-range which follows Kolmogorv's $-5 / 3$ scaling behavior. Results indicate an existence of Markovian properties at scales on the order of the Taylor micro-scale, $\lambda$ for most locations in the wake. The exception being directly behind the tips of the rotor and the hub where the complex turbulent interactions characteristic of the near-wake demonstrate influence upon the Markov process. The presence of a Markov process in the remaining locations leads to characterization of the multi-point statistics of the wind turbine wakes using the most recent states of the flow.
\end{abstract}




\section{Acknowledgements}

I would like to thank all of the individuals that have supported me during this pursuit. First, I must thank my friend and mentor Raúl Bayoán Cal for giving me the opportunity to pursue my curiosities. Thank you to Murat Tutkun for being patient and thoughtful while supplying guidance throughout this work. Thanks to Nicholas Hamilton and Betsy Camp for all of the support and inspiration. Thank you to Dr. Rectenwald and Dr. Zaron for acting as advisors during the final stages of this thesis. I would like to also thank my friends and family who have graciously accepted my commitment to further education and met it with enthusiasm.

Lastly I would like to thank my lovely wife Bonnie for for all of her continued love and support.

Sincerely, Matt 


\section{Contents}

Abstract i

Acknowledgements

List of Figures $\quad$ v

1 Introduction 1

1.1 Wind Power and Global Energy Demand . . . . . . . . . . . . . 1

1.2 Application of Markov Models to Wind Energy Analysis . . . . . . 4

1.3 Analysis of Wind Turbine Wakes . . . . . . . . . . . . . . 6

1.4 Thesis Outline ...................... 7

2 Theory 9

2.1 Identification of Turbulent Scales . . . . . . . . . . . . . . 10

2.2 Wilcoxon Rank-Sum Test . . . . . . . . . . . . . . . . . 16

3 Experimental Setup $\quad 19$

4 Results $\quad 24$

4.1 Mean Velocity Profile Data . . . . . . . . . . . . . 24

4.2 Wave Number Spectra . . . . . . . . . . . . . . . . 28 
4.3 Comparison of the 2-point and 3-point Conditional Probability . . . 30

4.4 Wilcoxon Test Results . . . . . . . . . . . . . . . . . 32

4.5 Local Reynolds number \& energy spectra . . . . . . . . . . . . . . . 40

4.6 Flattened Wave Number Spectra . . . . . . . . . . . . . . . . . . 42

5 Conclusions $\quad 44$

$\begin{array}{lll}6 & \text { Future Work } & 47\end{array}$

$\begin{array}{ll}\text { Bibliography } & 48\end{array}$ 


\section{List of Figures}

3.1 Side view of the wind tunnel experimental setup. . . . . . . . . . 20

3.2 Strakes to generate mean shear profile, from Cal et al, 2010 . . . . 21

3.3 Top view of model $3 \times 3$ array . . . . . . . . . . . . . . . . . 22

3.4 Schematic of streamwise and transverse measurement locations behind the middle turbine of the last row in model wind farm. . . . 23

4.1 Mean longitudinal velocity profiles for each measurement location. Data are organized by downstream location. The horizontal dashed lines represent the locations of the top and bottom tip of the rotor blade. . . . . . . . . . . . . . . . . 25

4.2 Turbulent Energy Production profiles for each measurement location. Data are organized by downstream location. The horizontal dashed lines represent the locations of the top and bottom tip of the rotor blade. . . . . . . . . . . . . . . . . . 26

4.3 Integral form of Kinetic Energy Flux profiles for each measurement location. Data are organized by downstream location. The horizontal dashed lines represent the locations of the top and bottom tip of the rotor blade. . . . . . . . . . . . . . . . . 28 
4.4 Normalized energy spectra for all vertical locations at 5 diameters downstream of the wind turbine. . . . . . . . . . . . . . 29

4.5 One dimensional conditional pdfs, from $x / D=3$, $p\left(v_{1}, r_{1} \mid v_{2}, r_{2} ; v_{3}=0, r_{3}\right)$ [blue] and $p\left(v_{1}, r_{1} \mid v_{2}, r_{2}\right)$ [red], where $\Delta r=\lambda$ and $v_{3}$ is conditioned to $0 \mathrm{~ms}^{-1} \ldots \ldots$. . . . . . 31

4.6 One dimensional conditional pdfs, from $x / D=3$, $p\left(v_{1}, r_{1} \mid v_{2}, r_{2} ; v_{3}=0, r_{3}\right)$ [blue], and $p\left(v_{1}, r_{1} \mid v_{2}, r_{2}\right)$ [red], where $\Delta r=0.3 \lambda$ and $v_{3}$ is conditioned to $0 \mathrm{~ms}^{-1} \ldots \ldots . \ldots 33$

4.7 Wilcoxon test results showing downstream trend at hub height, $y / D=1 \ldots \ldots \ldots \ldots \ldots \ldots \ldots$

4.8 Wilcoxon test results showing vertical location at top-tip, hub height and bottom-tip for $x / D=0.5$ (a, c, e) and $x / D=5$ (b,

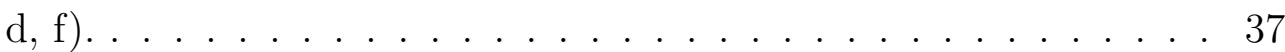

4.9 Spatial representation of Wilcoxon test results. . . . . . . . . . 39

4.10 Local Reynolds number based on the Taylor micro-scale, $R e_{\lambda}$, for each measurement location. . . . . . . . . . . . . . . . . 41

4.11 Local Taylor micro-scale, $\lambda$, for each measurement location . . . . . 42

4.12 Flattened energy spectra have been shifted vertically to highlight the inertial sub-range at critical locations in the wake $x / D=0.5$ at $y / D=1, x / D=2$ at $y / D=1.4, x / D=8$ at $y / D=1.75 \ldots 43$ 


\section{Chapter 1}

\section{Introduction}

\subsection{Wind Power and Global Energy Demand}

As the world energy demand continues to grow, the impact of energy production techniques will become ever more significant [1]. Currently the world's energy demands are primarily met through the burning of fossil fuels such as natural gas, coal, and petroleum [1]. Over the past two centuries the use of these fuels has provided the means for global industrialization and far reaching technological advances. The progress which accompanied the utilization of such fuel sources has until very recently out weighed the costs associated with the techniques. With the increasing negative effects of fossil fuel combustion on the environment in addition to limited stock of fossil fuel have forced many countries to inquire into and change to renewable energy sources to sustain the increasing energy demand [2]. Of primary concern over the use of fossil fuels is their limited quantity and non-renewable nature. Many estimates have been made and debated about when the decline of fossil fuels will begin $[3,4]$. Although such time lines are under debate, there is little doubt about the global impact that will follow. It is in response to these factors that demand for clean and renewable energy sources and techniques has moved to the forefront of 
international energy policies. Of the possible sources for such energy, wind energy has been identified as a resource which can offer a significant contribution to the world's future energy supply $[1,2,5]$.

Like all proposed solutions to the impending global energy crisis, wind energy is not without obstacles. Wind turbines operate in the atmospheric boundary layer and are thus subject to strong gradients of velocity and large velocity fluctuations in the form of gusts and atmospheric changes by time of day and season [6]. Geographic location of a wind farm is very important in order to maximize the productivity of a particular wind turbine or an entire turbine array. This leads to the second difficulty beset upon wind energy, locations that are considered to have a high wind resource are not always easily accessible. Often the locations that offer the greatest potential are situated offshore [7], or in distant rural areas, far away from the urban centers that would stand to benefit the most. This adds considerable cost to the installation of the wind farm and transmission of the power generated to nearby cities. Despite these obstacles the pursuit of wind energy continues. Since the 1990's the wind energy industry has been the fastest growing energy industry [2]. According to the most recent World Wind Energy Report, the total contribution of wind energy to the global supply is up to 3 percent, with a total of 282,275 megawatts (MW) [1].

To accompany the growth of the wind energy industry, the size of the turbine, blades, and the number of turbines used have also seen considerable growth. Today the largest turbines have a capacity of up to $7.6 \mathrm{MW}$ (Enercon, E-126) and a diameter of 154m (Siemens, SWT-6.0-154). Although most of the turbines used commercially have a capacity of 1-4 MW, they are now being used in large scale arrays, which can contain up to 290 turbines, as in the case of the Alta Wind Energy 
Center, an wind complex in California.

While wind energy has proven to be a significant source of renewable and clean energy, accurate projections of energy production are difficult to achieve [7-13]. Currently power projections are calculated using wind measurements collected at a met-mast outside of a given wind farm and at cup anemometers on the top of each turbine nacelle. For turbines which can manipulate the blade pitch, these measurements can be used to control the pitch of the turbine blades and direction the wind turbines face in order to maximize the power produced by the turbine [5]. The behavior of a single wind turbine wake has been studied extensively, but once a turbine is placed within an array the operating conditions can change considerably $[14,15]$. This is because, depending on their arrangement, turbines located downstream of another wind turbine experience an inflow of wind that includes the wake of the upstream turbine. The turbulent behavior within the internal boundary layer of a wind farm is influenced by turbine geometry, atmospheric conditions, and geographic features surrounding the farm. The interaction of these conditions creates an infinitely complex turbulent system which makes modeling the power production of a wind farm difficult. According to Barthelmie et al., power losses due to turbine wakes in large wind farms are predicted by state-of-the-art models to be of the order $10-20 \%$ of the total potential power output [16]. Among all of the values that can be measured, it has been shown that turbulence intensity within the internal boundary layer plays a significant role in the power output and mechanical fatigue of a wind turbine [8]. Therefore, effort is being put forth to predict the turbulence intensity throughout the internal boundary layer of a wind farm. 


\subsection{Application of Markov Models to Wind Energy Analysis}

In observing Markovian processes, characteristics of the turbulence become evident within the wind turbine canopy. Markov chain theory offers a valuable application to aid in the improved power production models. Markov theory states that if

the statistical development of a system can be determined, then the knowledge of the current state can provide enough information to determine the future state of the system; i.e. knowledge of the past states is not needed. Markovian statistical prediction methods have been applied in many ways, including multi-scale reconstruction of time series [17], to provide a diagnostic tool for identifying patients with congestive heart failure [18] and financial models [19-21].

Fundamental experimental studies have been conducted to describe the development of turbulent flows. Among other methods and studies, the statistics of small-scale turbulence are analyzed, searching for the existence of Markov processes. Markov processes have been discovered in several turbulent flows, such as a high Reynolds number axisymmetric jet [22], within the wake behind a cylinder [23], and fractal-generated grid turbulence [24]. In each of these studies, the flows demonstrated Markovian behavior for scale differences larger than or equal to the Taylor microscale, $\lambda$. Results from these studies also revealed that the extension of Markov process down to the Taylor microscale was independent of Reynolds number when $100<R e_{\lambda}<1000[22]$.

For wind farm applications, Markov models have been used to represent the degradation pattern of wind turbine components, as repair times are stochastic and turbine access is limited by weather conditions [10]. The use of partially observed Markovian decision process has been suggested as a way to improve maintenance 
scheduling [10]. A Markov model for the performance of wind turbines that accounts for component reliability, the effect of wind speed, and turbine capacity was found to predict repair schedules with an accuracy of $10 \%$ or better when the number of turbines is greater than approximately 100 [9].

Application of Markovian theory to atmospheric turbulence was addressed as a way to predict power production of a wind turbine by Anahua et al. [8]. Anahua et al. showed that even with different turbulence intensities, the characteristic of the wind turbine power performance can be properly reconstructed. The method was not only more accurate than the standard procedure of ensemble averaging but it also allowed a faster and robust estimation of wind turbines' power curves. Their study addressed the turbulent fluctuations experienced by a wind turbine, which are not dealt with correctly by use of ensemble averaging [8].

Current control methods and fatigue models often use field data collected in 10 minute ensemble averages to predict power production or maintenance schedules. The use of 10 minute ensemble average predictive models are quite accurate when applied to a single wind turbine. However, there are significant discrepancies between the models and the experimental results once the turbines are interacting with the wakes of other turbines. Research has shown that wind turbines operating within an array have a power generation loss of up to 40 percent, when compared to a freestanding wind turbine $[12,13]$. Ma et al. used these findings to show that the total power output of all wind turbines in a wind farm is not a simple aggregation of the power that can be expected from individual turbines [11]. 


\subsection{Analysis of Wind Turbine Wakes}

Wind turbine wakes are commonly analyzed by examining one of two regions, namely the near-wake and the far-wake. Near-wake region extends up to one diameter downstream from the rotor, where the flow field is strongly influenced by the local motion of the rotor blades. In this region the flow is influenced by the number of blades and blade aerodynamics, including stalled flow, tip vortices, and 3-D effects [25]. After some distance downstream of the rotor, the near wake structures break down and as this process happens the wake is said to transition to the far-wake region. The far-wake is generally characterized by two mechanisms, advection and diffusion. The interest in this region pertains to situations where the wind turbines exist in clusters, cf. Vermeer et al. [15]. The analysis shows that due to the inflow condition dependency on the wake of an upstream turbine, it is necessary to investigate the statistical behavior of the wake in order to improve the methods for controlling power production.

Porté-Agel et al. studied the effects of surface roughness on the wake area and flow characteristics, demonstrating the sensitivity of turbine wakes to environmental parameters and inflow conditions of the atmospheric boundary layer [26]. Cal et al. showed that the vertical flux of kinetic energy is of the same order of magnitude as the power extracted by the wind turbines, thus highlighting the importance of vertical transport in the boundary layer [27].

Vermeer et al. performed a survey of available experimental and numerical data in both the near and far-wake regions, showing a fully developed turbulent flow after several rows of turbines [15]. This concept was verified in an experimental wind farm study, where Chamorro et al. found that below the top tip, turbulence statistics 
appear to reach equilibrium as close as the third to fourth row of wind turbines [28]. This "fully developed" state of a wind turbine array boundary layer leads to what is being considered an infinite array condition where the wake statistics beyond this point are considered to be representative of the corresponding points within an array and no longer change no matter how large the array is. This infinite array concept gives way to a few assumptions that can be used in wind tunnel experiments, allowing for data collected in small scale arrays of four rows or more to represent an infinite array.

Markov property analysis is applied to the turbulent boundary layer in a wind turbine array. Hot-wire anemometry signals within the array are analyzed to determine the existence and limitations of Markov properties in the wake. The results are discussed in the context of turbulence theory and wind turbine wake development.

The turbulence within a wake of a wind turbine is shown to exhibit Markovian properties for scales on the order of the Taylor microscale at many locations. Locations in the near-wake demonstrate scale dependance for the Markov process which is attributed to the geometric influence of the wind turbine and periodic structures caused by the rotation of the blades such as tip vortices. Points outside of the near-wake lose the scale dependence demonstrated in the near-wake, as the instability of the turbulence cascade breaks up the near-wake structures.

\subsection{Thesis Outline}

The presence of Markov processes in fundamental turbulent systems such as a roundjet, grid generated turbulence, and turbulent boundary layers has lead to accurate 
predictability of the velocity distributions in these systems. The question this work addresses is whether or not the turbulent internal boundary layer of a wind turbine array demonstrates Markovian statistical behavior despite the complexity of the flow. The goal of this research is to identify the locations in the wake of a wind turbine that demonstrate Markovian behavior. Once the extent of the Markov process is identified, the results will be compared to quantities of Reynolds number, turbulent kinetic energy production, and vertical kinetic energy flux. The theory to be applied herein will be outlined in Chapter 2, Chapter 3 provides details on the experimental setup, results from the experiment are presented in Chapter 4, conclusions are given in Chapter 5, and future work is discussed in Chapter 6. 


\section{Chapter 2}

\section{Theory}

In 1941, Kolmogorov described turbulent flow as a combination of a wide range of scales of motion, or so-called eddies, within the flow [29]. At sufficiently high Reynolds numbers, development of turbulent eddies can be divided into three ranges. The first range defined as the energetic range is said to be the largest scales present in the flow, often referred to as integral scales, which contain the kinetic energy associated with the flow. The largest scales are determined by the boundary and initial conditions of the system. Due to the nature of high Reynolds number flows, these scales are independent of viscosity. Furthermore, the kinetic energy is then transferred from large scale to smaller scale eddies through non-local interactions.

Once the energy has moved to an eddy of sufficiently small scale, viscosity begins to play a role. At the smallest scales the kinetic energy is transferred into internal energy through viscous dissipation, thus these smaller scales are referred to as dissipative scales [29]. Kolmogorov suggested that in between the integral scales and

dissipative scales there exists a series of intermediate scales which are considered to be, at sufficiently high Reynolds numbers, locally isotropic. This third range is referred to as the inertial range of turbulent scales.

Through the use of scaling analysis Kolmogorov was able to show that within the 
inertial range, the spectra follow a $-5 / 3$ power law as energy is moved from larger to smaller scales. It is therefore important to define the integral and dissipative scales present in the system so as to define the range of scales that would fall within the inertial range. Within the turbulent flow, the integral scales are characterized by low wave numbers, which have units of $1 / m$, conversely the smallest scales have very large wave numbers. Within a turbulent system the small scales are nested within the integral scale motion and are correlated to the behavior of energetic scales. To do identify the integral and dissipative length scales it is necessary to analyze the auto-correlation coefficients of the instantaneous velocity signal.

\subsection{Identification of Turbulent Scales}

In many turbulent flows, the statistical properties of the process are independent of origin in time, and if that is the case, the process is said to be stationary. When dealing with a stationary random process one of the most useful statistical quantities is the auto-correlation defined as the average product of the random variable measured at two different times,

$$
C(\tau)=\langle u(t) u(t+\tau)\rangle
$$

where $u(t)$ is an observed instantaneous velocity at time $t$ and $\tau$ is the time interval between the two measurements and $\langle 0\rangle$ denotes a suitably-defined average. The auto-correlation function describes the "memory" of the process, that is the time over which the process is correlated to itself [30]. For a stationary random process given a large enough time interval, $\tau$, the value of the auto-correlation will reach zero 
indicating a loss of correlation. Some important properties of the auto-correlation function are that it is symmetric about $\tau=0$; that is,

$$
C(\tau)=C(-\tau)
$$

and the maximum value of correlation is found at $\tau=0$ indicating perfect correlation. It is useful to normalize the auto-correlation function by the maximum value, the results referred to the auto-correlation coefficient given by:

$$
\rho(\tau)=\frac{C(\tau)}{C(0)}=\frac{\langle u(t) u(t+\tau)\rangle}{\left\langle u(t)^{2}\right\rangle} .
$$

To determine the range of time over which the process is correlated with itself the function is integrated over the total time interval:

$$
T_{\mathrm{int}}=\int_{0}^{\infty} \rho(\tau) d t
$$

which gives the integral time scale for the given process.

In order to convert this time scale into a physical length scale one can use Taylor's hypothesis of turbulent frozen fields. According to Taylor, "If the velocity of the airstream which carries eddies is much greater than the turbulence velocity, one may assume that the sequence of changes in $u$ at a fixed point are due to the passage of an unchanging pattern of turbulent motion over the point" [31]. Therefore the time scales calculated here can be transformed into spatial values by multiplying by the convective velocity, giving physical size to the eddies within the system. Thus to find the integral length scale, $L$, multiply the value for $T_{\mathrm{int}}$ by the mean convective velocity, $U$ : 


$$
L=T_{\text {int }} U
$$

gives a physical length of the largest eddies present in the system. The integral length scale can also be approximated by,

$$
L_{\infty}=0.9 \frac{\left(u^{\prime}\right)^{3}}{\epsilon}
$$

as proposed by Mydlarski and Warhaft [32] for isotropic flow, where $\epsilon$ is the rate of dissipation and $u^{\prime}$ is the velocity fluctuation about the mean convective velocity $\mathrm{U}$. The rate of dissipation is defined as

$$
\begin{gathered}
\epsilon=2 \nu\left\langle s_{i j} s_{i j}\right\rangle \\
s_{i j}=\frac{1}{2}\left(\frac{\partial u_{i}^{\prime}}{\partial x_{j}}+\frac{\partial u_{j}^{\prime}}{\partial x_{i}}\right) .
\end{gathered}
$$

In the case of isotropic turbulence, originally outlined by Taylor [33], it can be shown that dissipation can be estimated as

$$
\epsilon=15 \nu\left\langle\left(\frac{\partial u^{\prime}}{\partial x}\right)^{2}\right\rangle
$$

Using this estimate for the rate of dissipation, the smallest physical length scales, which are responsible for the dissipation of kinetic energy can be estimated. These length scales are characterized by Kolmogorov micro-scale, which is defined as,

$$
\eta_{k}=\left(\frac{\nu^{3}}{\epsilon}\right)^{\frac{1}{4}}
$$


In the atmosphere, where the integral scales can be on the order of a kilometer, the Kolmorogov micro-scale can be found to be on the order of $1-10 \mathrm{~mm}$ [30]. To evaluate the separation of scales the ratio of $L_{\infty}$ to $\eta_{k}$ is evaluated, $L_{\infty} / \eta_{k}>10^{4}$. If the ratio is less than $10^{4}$ then the values calculated are considered to be approximate.

As stated above, Kolmogorov's length scale is used to characterize the smallest measured scale of turbulence in the flow. However it does not identify scale at which the non-linear behavior of dissipation begins, marking the end of the inertial range. This scale was identified by Taylor [33], and is hence referred to as the Taylor micro scale.

Based on the auto-correlation function, the Taylor micro-scale, $\lambda$, can be obtained. Using the auto-correlation function, this scale is estimated through using the first non-zero value of the series expansion which describes the auto-correlation curve. Another approach is to use the average distance between the zero crossings of the osculating parabola of the auto-correlation function over large time intervals, or more explicitly obtained by,

$$
\lambda^{2}=\frac{\left\langle u(t)^{2}\right\rangle}{\left\langle\left(\frac{\partial u}{\partial t}\right)^{2}\right\rangle} .
$$

The interaction of scales is represented mathematically by the nonlinear term of the Navier-Stokes equation. Describing this interaction requires multipoint statistics in order to fully capture the behavior of the cascade. By analyzing the simultaneous interaction of all of the scales in a turbulent flow, it is theoretically possible to create a complete statistical description of the cascade. Through the application of Markov chain theory it becomes possible to evaluate the statistical behavior of the turbulent system as a diffusion process. 
Statistics of velocity increments are used to characterize the turbulent cascade in physical space. The longitudinal velocity increments are considered for this analysis and are described as,

$$
v(r, t)=u^{\prime}(x+r, t)-u^{\prime}(x, t)
$$

where $v$ and $u$ represent velocity increment and velocity fluctuation respectively. The spatial distance between the two measurements is denoted by $r$, while $x$ represents the origin. Due to the stationarity of the statistical properties of the smallest scales, the dependence upon time is omitted in the formulation. Kolmogorov's theory predicts $n^{t h}$ - order structure function, $\left\langle v(r)^{n}\right\rangle$ to be strictly a function of $r$ and $n$, where $\left\langle v(r)^{n}\right\rangle \sim r^{n / 3}[29]$. Also, when $n=2$, the second order structure function, $\left\langle v(r)^{2}\right\rangle$ quantitatively describes the distribution of turbulent kinetic energy over different scales defined by the spatial separation, $r$ [24].

One way of studying moments of velocity increments is to calculate the probability density function (pdf) of the velocity increments at different scales, $p(v, r)$. Since a complete description of the turbulence cascade is only possible by taking into account the interaction of all scales of motion, a multi-scale (N-point) joint pdf, $p\left(v_{1}, r_{1} ; v_{2}, r_{2} ; v_{3}, r_{3} ; \ldots ; v_{n}, r_{n}\right)$ must be constructed.

An essential quantity used in utilizing the theory of Markov processes is the conditional probability density functions. Define a joint pdf, $p\left(v_{1}, r_{1} ; v_{2}, r_{2}\right)$, used to find the probability of $v_{1}$ occurring at scale difference $r_{1}$ and $v_{2}$ occurring at scale difference $r_{2}$, where $r_{1}<r_{2}$. One can find the conditional probability of $v_{1}$ occurring at scale difference $r_{1}$, given that velocity $v_{2}$ is occurring at scale difference $r_{2}$ by using the definition of a conditional probability, 


$$
p\left(v_{1}, r_{1} \mid v_{2}, r_{2}\right)=\frac{p\left(v_{1}, r_{1} ; v_{2}, r_{2}\right)}{p\left(v_{2}, r_{2}\right)}
$$

This technique can be extended to all present velocity increments and scale differences within a respective system. For instance, to find the conditional pdf of $v_{1}$ at $r_{1}$ conditioned by all velocities and scales present, the conditional pdf is defined as $p\left(v_{1}, r_{1} \mid v_{2}, r_{2} ; \ldots ; v_{n}, r_{n}\right)$. To find the conditional pdf for this case, joint pdfs can be used and given by,

$$
p\left(v_{1}, r_{1} \mid v_{2}, r_{2} ; \ldots ; v_{n}, r_{n}\right)=\frac{p\left(v_{1}, r_{1} ; v_{2}, r_{2} ; \ldots ; v_{n}, r_{n}\right)}{p\left(v_{2}, r_{2} ; \ldots ; v_{n}, r_{n}\right)}
$$

Obtaining an N-point conditional pdf provides a complete velocity increment scale relation throughout the cascade. This is particularly difficult to do in practice, due to the large number of scales that exist in a turbulent flow field.

Applicability of Markov's theory offers a significant simplification in the calculation of the N-point conditional pdf. If the system is Markovian then the probability of the current state can be fully determined by the most recent state. Thus, the N-point conditional pdf of the velocity increments with Markovian properties can be simplified as follows:

$$
p\left(v_{1}, r_{1} \mid v_{2}, r_{2} ; \ldots ; v_{n}, r_{n}\right)=p\left(v_{1}, r_{1} \mid v_{2}, r_{2}\right)
$$

From there, N-point joint pdf of the process can be constructed in terms of multiplication of the conditional pdfs as, 


$$
p\left(v_{1}, r_{1} ; v_{2}, r_{2} ; \ldots ; v_{n}, r_{n}\right)=\prod_{i=1}^{N-1} p\left(v_{i}, r_{i} \mid v_{i+1}, r_{i+1}\right) p\left(v_{n}, r_{n}\right)
$$

The implication of equation (2.16) is that knowledge of a conditional pdf $p\left(v_{i}, r_{i} \mid v_{i+1}, r_{i+1}\right)$ for any two scales $r_{i}$ and $r_{i+1}$ is enough to reconstruct the complete statistical description of the velocity increments embedded in the N-point pdf, $p\left(v_{1}, r_{1} ; v_{2}, r_{2} ; \ldots ; v_{n}, r_{n}\right)$. In order for the process to be considered Markovian, equation (2.15) must be satisfied. It is clearly difficult to analyze all present scales of turbulence, however with a data set of $4 \times 10^{6}$ measurements it is possible to examine the joint probabilities up to values of $n=3$. Thus, equation (2.15) is transformed and expressed as:

$$
p\left(v_{1}, r_{1} \mid v_{2}, r_{2} ; v_{3}, r_{3}\right)=p\left(v_{1}, r_{1} \mid v_{2}, r_{2}\right)
$$

A comparison of the two and three point conditional pdfs is conducted to evaluate the similarity between the probabilities in an effort to determine the Markovian nature of the process. The process of comparing overlapping conditional probabilities gives qualitative evidence to the existence of a Markov process.

\subsection{Wilcoxon Rank-Sum Test}

In order to create a quantitative understanding of whether two conditional pdfs are equal, the Wilcoxon rank-sum test is employed. The result of the Wilcoxon test identifies the scales and scale differences at which two stochastic variables $(a$ and $b$ ) have the same probability distributions $[17,21,23]$. The test begins with 
computing the total number of inversions, $Q$, after samples from $a=\left[a_{1}, a_{2}, \ldots, a_{m}\right]$ and $b=\left[b_{1}, b_{2}, \ldots, b_{n}\right]$ are merged as well as sorted in ascending order. An inversion occurs when the information in the merged data set switches from the information of one data set to the other. For example, if the two stochastic variables $a$ and $b$ are combined into a new data set $c$ and sorted in ascending order as shown:

$$
c=\left[a_{1}, a_{2}, b_{1}, b_{2}, a_{3}, b_{3}, b_{4}, a_{4}, \ldots\right]
$$

There is an inversion between $a_{2}$ and $b_{1}, b_{2}$ and $a_{3}$, and so on giving four inversions in this example.

For a large sample size of $m, n>25$, as is the case for measurements reported in this thesis, it is assumed that $Q$ is normally distributed with a mean value

$$
\langle Q\rangle=\frac{m n}{2}
$$

and a standard deviation of

$$
\sigma_{q}=\sqrt{\frac{m n(m+n+1)}{12}} ;
$$

assuming the equality $p(a)=p(b)$ holds. The absolute value of the standardized variable is

$$
\Delta Q=\frac{|Q-\langle Q\rangle|}{\sigma_{q}} .
$$

This is found to be a half-normal distributed random variable with a mean of $\sqrt{2 / \pi}$. 
Dividing the standardized variable $\Delta Q$ by its mean yields:

$$
\left\langle\Delta Q^{*}\right\rangle=\frac{|Q-\langle Q\rangle|}{\sigma_{q} \sqrt{2 / \pi}}
$$

If the relationship $p(a)=p(b)$ is satisfied, then the measured value for $\left\langle\Delta Q^{*}\right\rangle$ will be unity. It follows that any departure from unity indicates the level of inequality between probabilities $p(a)$ and $p(b)$. The statistical methods described above are used in the subsequent sections to characterize the wake of a model wind turbine array boundary layer as a Markov process. When applied to the two and three-point conditional pdfs from Equation 2.17, a value of $\left\langle\Delta Q^{*}\right\rangle=1$ indicates that the process is Markovian for the scales within the density functions. 


\section{Chapter 3}

\section{Experimental Setup}

The experiment was conducted in the Corrsin Wind tunnel at The Johns Hopkins University. The test section of the wind tunnel is $10 \mathrm{~m}$ long, $0.9 \mathrm{~m}$ high, and $1.2 \mathrm{~m}$ wide. The wind tunnel is a closed-loop return type, with two contractions, a 25:1 primary and a 1:1.27 secondary. To replicate features of the atmospheric boundary layer in which wind turbine arrays commonly encounter, the inflow must be conditioned. The inflow velocity profile is shaped using strakes, an active grid, as well as a rough surface composed of sandpaper to generate a mean shear profile and freestream turbulence. The active grid in this wind tunnel is used to introduce high levels of free-stream turbulence. The design of the active grid follows the design criteria outlined in Refs. [32,34] and consists of seven vertical and five horizontal rotating aluminum shafts. Individual shafts rotate independently and are driven by a $1 / 4 h p$ AC motor. Each motor is actuated independently and set to rotate at random speed and directions, changing every second within a range of 210 and $420 \mathrm{rpm}$. Each horizontal and vertical shaft has eight and six winglets respectively. Each winglet is a $10 \times 10 \mathrm{~cm}^{2}$ square made of $3.18 \mathrm{~mm}$ thick aluminum. Each plate has several $20 \mathrm{~mm}$ diameter circular cutouts.

Strakes are used to shear the mean velocity profile at the inlet. Nine strakes are 


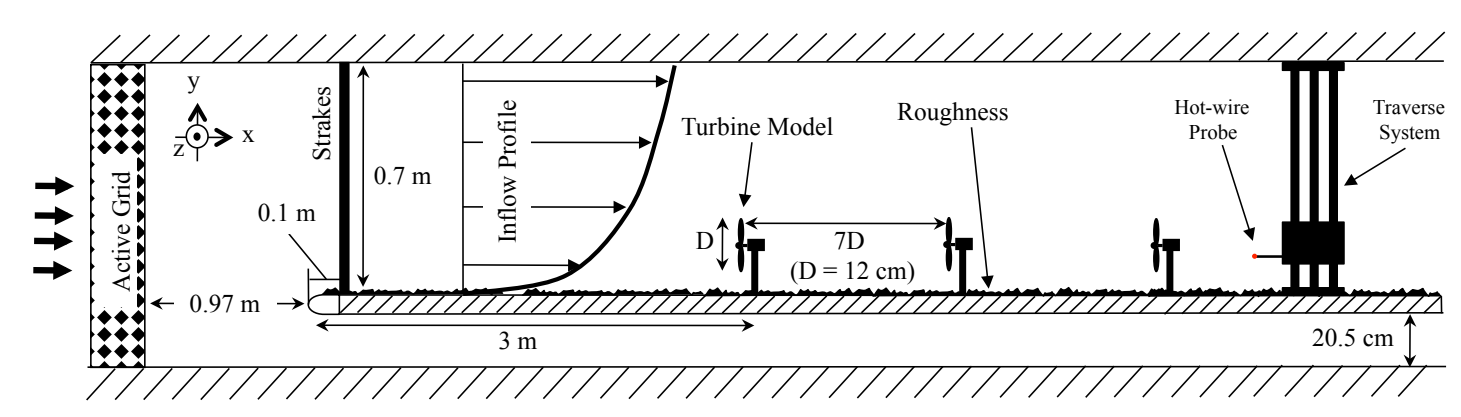

Figure 3.1: Side view of the wind tunnel experimental setup.

equally distributed across the test section normal to the primary flow direction as shown in Figure 3.2. Each strake is laser cut from $12.7 \mathrm{~mm}$ thick acrylic. The blockage of the strakes decreases with wall normal distance in a piecewise linear fashion. This shape results in the desired velocity profile and turbulence intensity.

The strakes were positioned $0.1 \mathrm{~m}$ downstream of the leading edge of an elevated flat plate. This plate was used to generate a fresh boundary layer within the test section. The plate is $6.7 \mathrm{~m}$ long and $19 \mathrm{~mm}$ thick and spans the width of the tunnel test section. The leading edge is located $0.97 \mathrm{~m}$ downstream of the active grid and has an elliptical leading edge with a 3:1 profile. The top side of the flat plate is covered with 24-grit aluminum oxide sand paper.

A $3 \times 3$ array of model wind turbines with a rotor diameter, $D$, of $12 \mathrm{~cm}$ and hub height of $12 \mathrm{~cm}$ were placed on the top of the elevated flat plate $3 \mathrm{~m}$ downstream of the leading edge. Three bladed rotors of $D=12 \mathrm{~cm}$ diameter are cut from $0.48 \mathrm{~mm}$ sheet metal. Each blade was twisted from $15^{\circ}$ at the root to $10^{\circ}$ at the tip. The twist was measured from the plane normal to the primary flow direction and were adjusted slightly until the desired angular velocity was reached that corresponded 


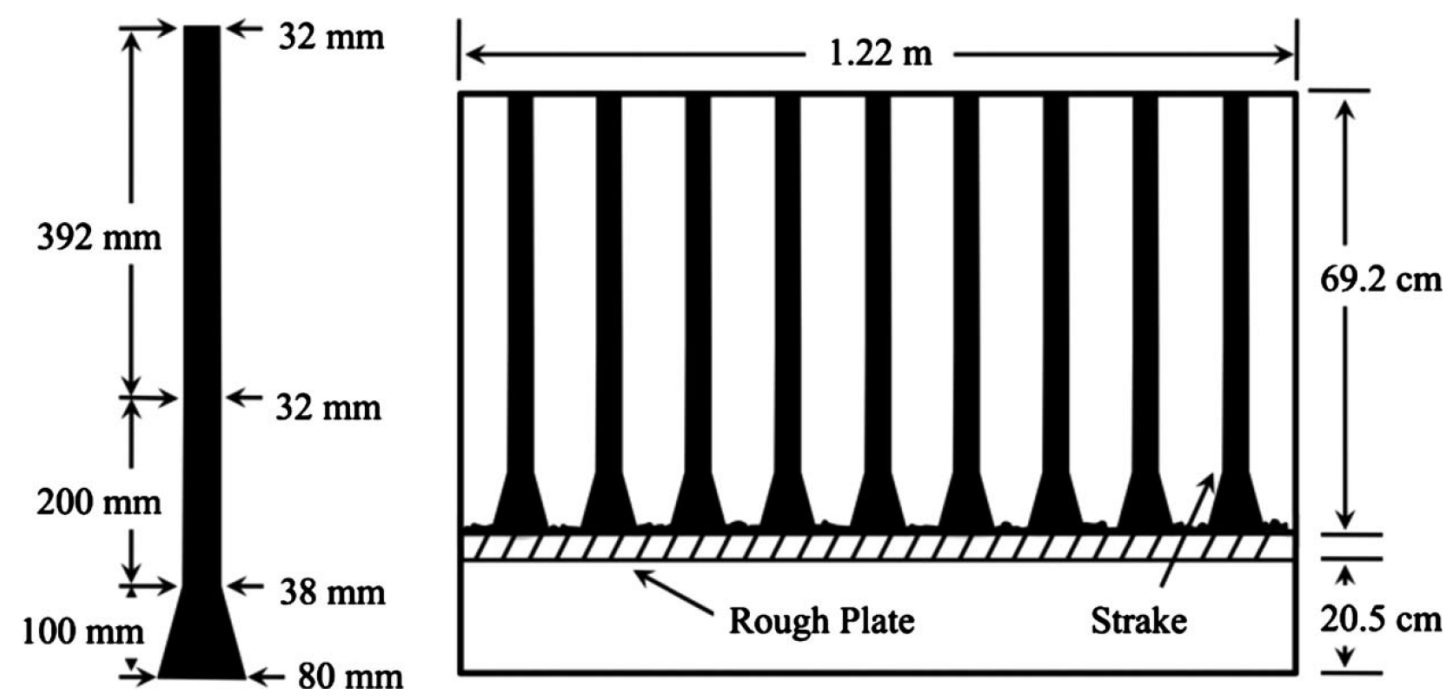

Figure 3.2: Strakes to generate mean shear profile, from Cal et al, 2010

to the tip speed ratio seen in real world turbine arrays. The towers have a $1 \mathrm{~cm}$ diameter, are $10.7 \mathrm{~cm}$ tall and were made using rapid prototyping. When the model nacelle is placed onto of the tower the hub height of the rotor is $12 \mathrm{~cm}$.

A schematic of the setup is shown in Figure 3.1. A reference mean velocity of $9.4 \mathrm{~ms}^{-1}$ was selected as a characteristic wind velocity. Each turbine column was spaced $3 D$ from the next in the cross-stream direction and each row was spaced $7 D$ down stream from the preceding row. The downstream spacing is sufficient that the wake statistics past the third row of turbines are representative of an infinite array [26].

Using X-type hot-wire anemometry to collect data, velocity measurements were taken at a sampling frequency of $40 \mathrm{kHz}$ for 100 seconds at each location of the sample set. The wire dimensions are $500 \mu \mathrm{m}$ in length and $5 \mu \mathrm{m}$ in diameter. To characterize the inflow conditions, several point measurements were conducted in 


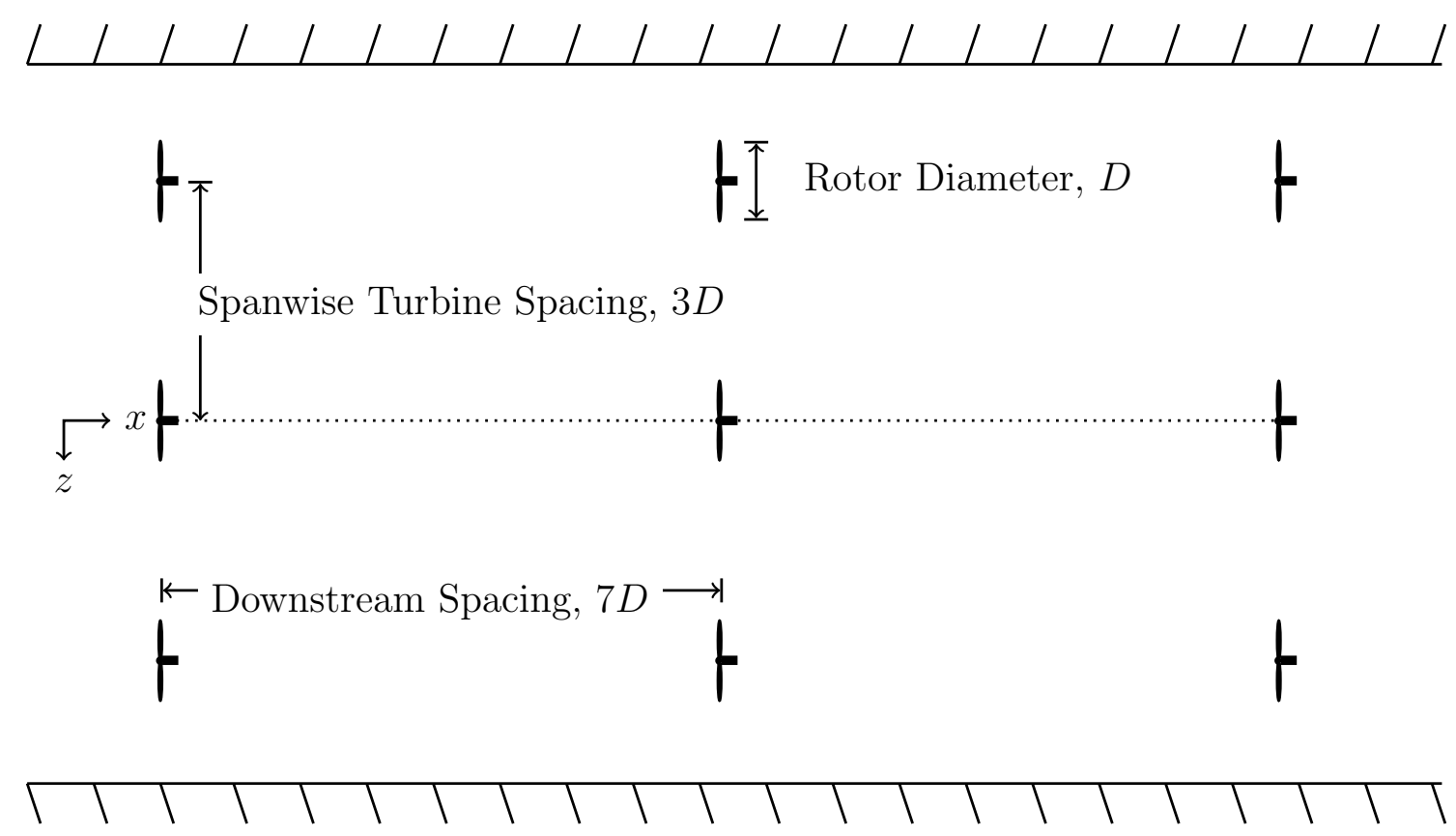

Figure 3.3: Top view of model 3 x 3 array

increasing distance from the elevated flat plate surface, ranging from 0.005 to $0.48 \mathrm{~m}$. Hot-wire measurements were collected at $2.9 \mathrm{~m}$ from the leading edge of the plate, $0.1 \mathrm{~m}$ upstream of the wind turbine array. Mean velocities and turbulent fluctuations are measured for all three spatial directions. The mean velocity and Reynolds stress profiles were examined along the wall normal direction to verify the shape of the incoming boundary layer, [27]. This process is repeated for the span wise direction at a fixed streamwise and wall normal coordinate, over a range of $z=-0.24$ to $0.24 \mathrm{~m}$ at $2 \mathrm{~cm}$ increments from the center line, revealing reasonable uniformity [27].

The sampling set includes one streamwise - wall-normal (XY) plane, behind the middle row wind turbine located in the last row of the array. The plane extends from the exit of the turbine to $8 D$ downstream, measurements were taken $1 \mathrm{~cm}$ increments in wall-normal direction at 21 locations starting at $0.5 \mathrm{~cm}$ above floor at downstream 
increments of $1 D$, as shown in Figure 3.4. Measurements were initially collected for wall-normal and streamwise velocities, then the probe was rotated to collect data for the cross-stream and downstream velocities. Each filled circle indicates a location where 21 measurements along the wall-normal direction were made.

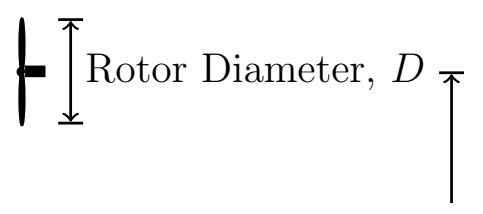

Spanwise Turbine Spacing, 3D
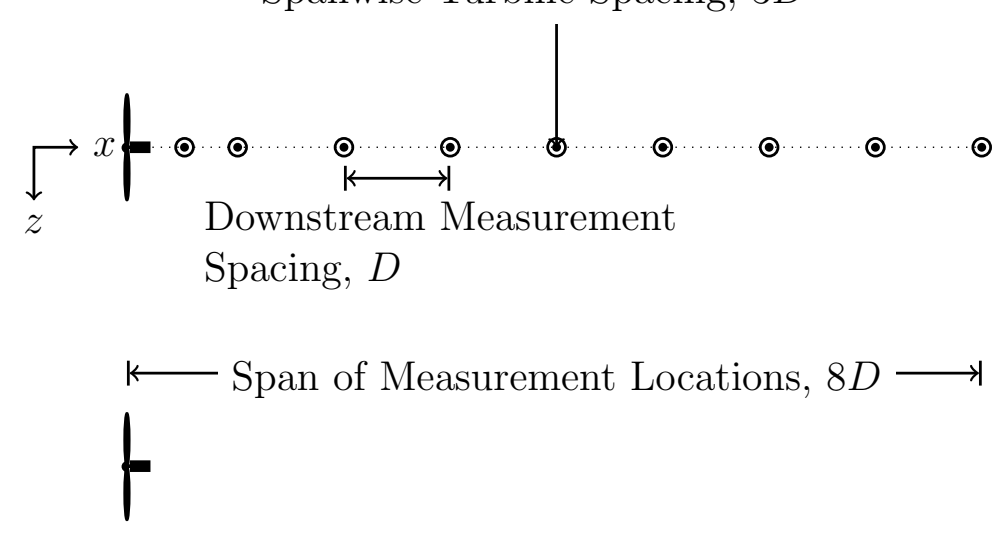

Figure 3.4: Schematic of streamwise and transverse measurement locations behind the middle turbine of the last row in model wind farm.

To estimate the integral length scale, point measurements from directly above the last turbine at $y / D=1.75$ are used to calculate the scales using equations 2.6 and 2.5. The results of the computations showed that $L_{\infty}=0.158 \mathrm{~m}$ and $L=0.173 \mathrm{~m}$, revealing that the flow at small scales is nearly isotropic. The Kolmogorov microscale is calculated using the assumption of isotropic small scale turbulence with a resulting value of $2.14 \times 10^{-4} \mathrm{~m}$ The ratio of $L_{\infty}$ and $\eta_{k}$ was found to be 740 which falls within the range of values found by Mydlarski and Warhaft [32] indicating the existence of an inertial range. 


\section{Chapter 4}

\section{Results}

In the absence of the atmospheric boundary layer, a wind turbine wake is in itself a highly complicated system. Contained within the near-wake of a wind turbine wake, the flow is marred by complexity including tip vortices, stalled flow and threedimensional effects caused by the rotating blades [15]. The standard is to space turbines 5-7 diameters downstream of each other to reduce the impact of these near-wake structures on the preceding turbines. More tightly arranged farms suffer due to the close proximity spacing leading to higher levels of turbulence intensity experienced by downstream turbines.

In field measurements it is important to note that most 'tracking/maintenance' measurements are done at the nacelle. Nevertheless, the characteristics of the wake are highly dependent upon the location of the probe, both in the longitudinal, $x / D$, and the vertical, $y / D$, locations.

\subsection{Mean Velocity Profile Data}

To characterize the flow present behind a wind turbine within an array, the mean longitudinal velocity profiles, turbulent kinetic energy production and kinetic energy flux are analyzed. The data plotted in Figure 4.1 contains the mean longitudinal 


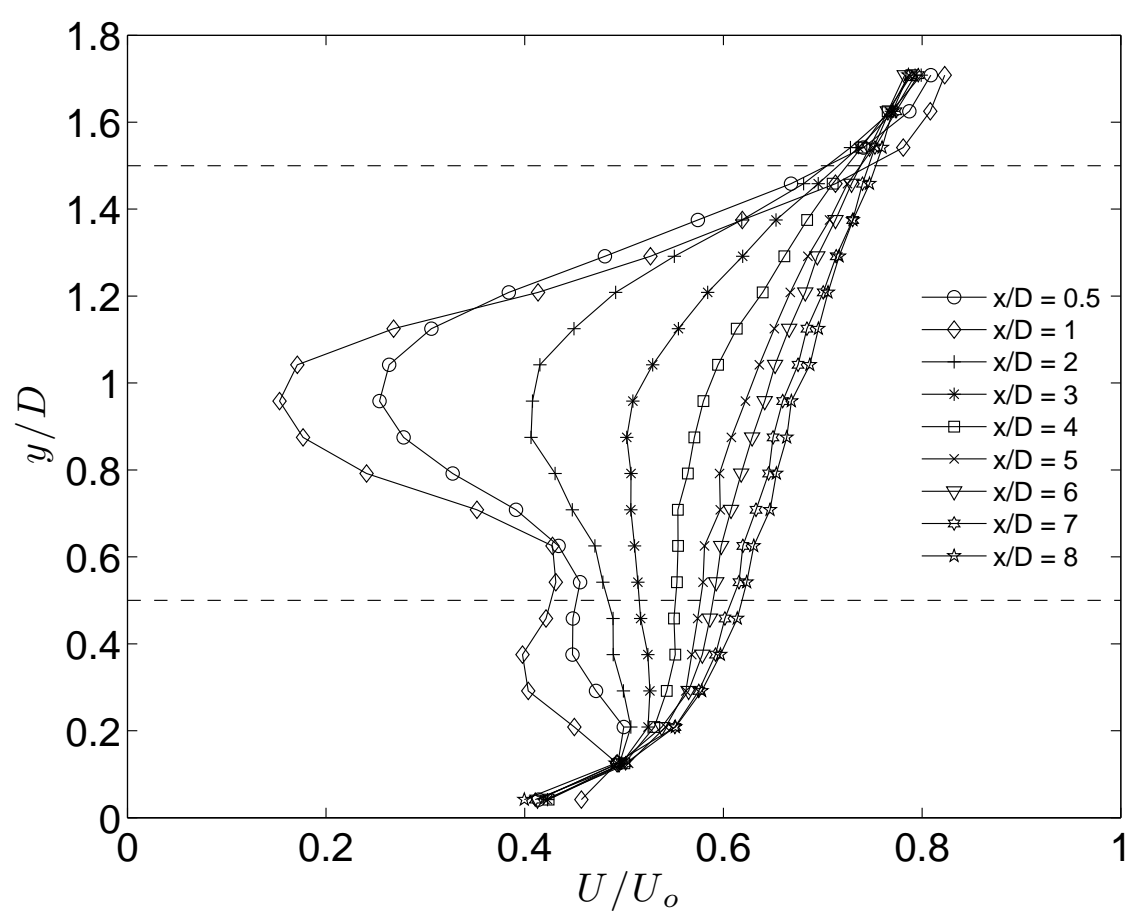

Figure 4.1: Mean longitudinal velocity profiles for each measurement location. Data are organized by downstream location. The horizontal dashed lines represent the locations of the top and bottom tip of the rotor blade.

velocity, $U$, which is normalized by the reference mean velocity of the wind tunnel, $U_{o}=9.4 \mathrm{~m} / \mathrm{s}$. The figure presents nine wall-normal profiles, one for each $x / D$ location. The dotted horizontal lines represent the top and bottom tip of the turbine rotor. The results show that the velocity deficit is most pronounced in the near-wake region as expected. By $x / D=5$, the velocity profile has recovered and increases uniformly, which indicates the existence of the far-wake region [35]. These results are similar to previous studies that addressed wind turbine wake recovery $[25,27,28]$.

Next, the production of turbulent kinetic energy, $-\left\langle u^{\prime} v^{\prime}\right\rangle \partial U / \partial y$, is plotted versus the wall-normal coordinate. These are normalized by $U_{o}^{3} / D$ and $D$, respectively. As 


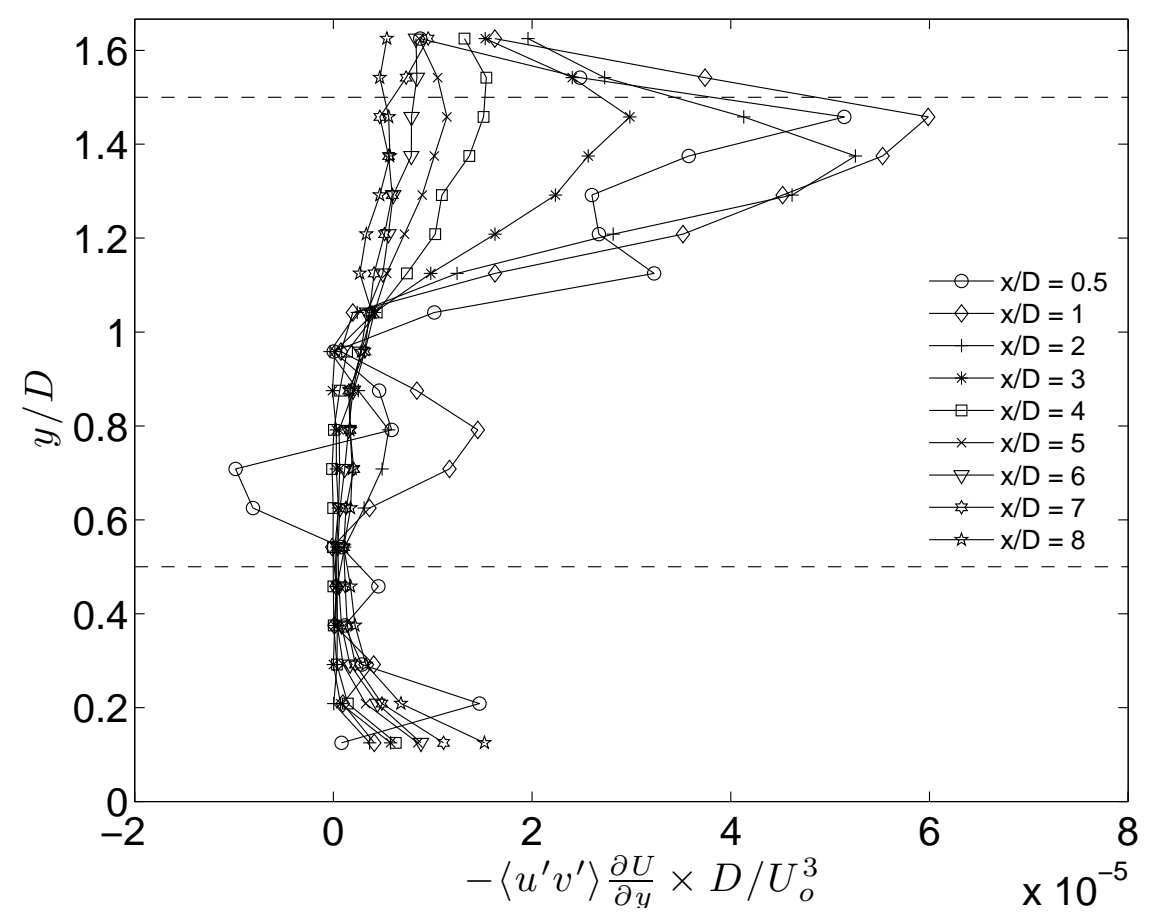

Figure 4.2: Turbulent Energy Production profiles for each measurement location. Data are organized by downstream location. The horizontal dashed lines represent the locations of the top and bottom tip of the rotor blade.

shown in Figure 4.2, the locations near the top tip of the rotor show the largest values production. As the downstream distance from the rotor increases, the values for production become negligible. There is a noticeable change in the production values at $x / D=4$, where the peak values around the top tip drop by $50 \%$. Production at the measurement locations nearest to the floor is shown to increase with $x$, indicating the effect of the wall where a boundary layer exists and the wall gradients are dominant as well as the top tip of the rotor for the longitudinal location range of $x / D=0.5-4$.

The kinetic energy flux, $\partial\left(-\left\langle u^{\prime} v^{\prime}\right\rangle U\right) / \partial y$, has been shown to play a significant role 
in the process of energy replacement within the wakes of wind turbines. Cal et al. were able to show that the vertical fluxes of mean kinetic energy associated with the streamwise velocity due to Reynolds turbulent shear stresses are of the same order of magnitude as the power extracted by the wind turbines [27]. The vertical flux of mean kinetic energy is therefore an important quantity for analyzing the process of energy extraction from the flow to the turbine as well as wake remediation and downstream development. More importantly, in the near-wake region, the effect of the rotor at the top tip is to generate the largest magnitudes of kinetic energy flux.

Figure 4.3, shows the integral from of the kinetic energy flux is normalized by the cube of $U_{o}$ then plotted for each measurement position, grouped by respective $x / D$ locations. The difference between the top tip and bottom tip values decreases as the downstream location $x / D$ increases. This is a result of the large quantities of energy being pulled from the free stream to replenish the momentum deficit created by the turbine. As the wake remediates, the flux near the bottom tip becomes relatively small and slowly increases with $\mathrm{x}$. It is once again noticeable that at $x / D=4$ the profiles drastically change in shape with a rather monotonic behavior in the wallnormal direction. The values at the bottom tip nearest to the rotor, $x / D=0.5$ and $x / D=1$, do not follow the same trends as the other profiles due to a direct effect of the turbine mast on the mean velocity profile, which is also reflected in Figure 4.1. Thus, these results provide context for the behavior in which Markovian properties can be observed and regions where Markovianity is predominant at a particular scale. 


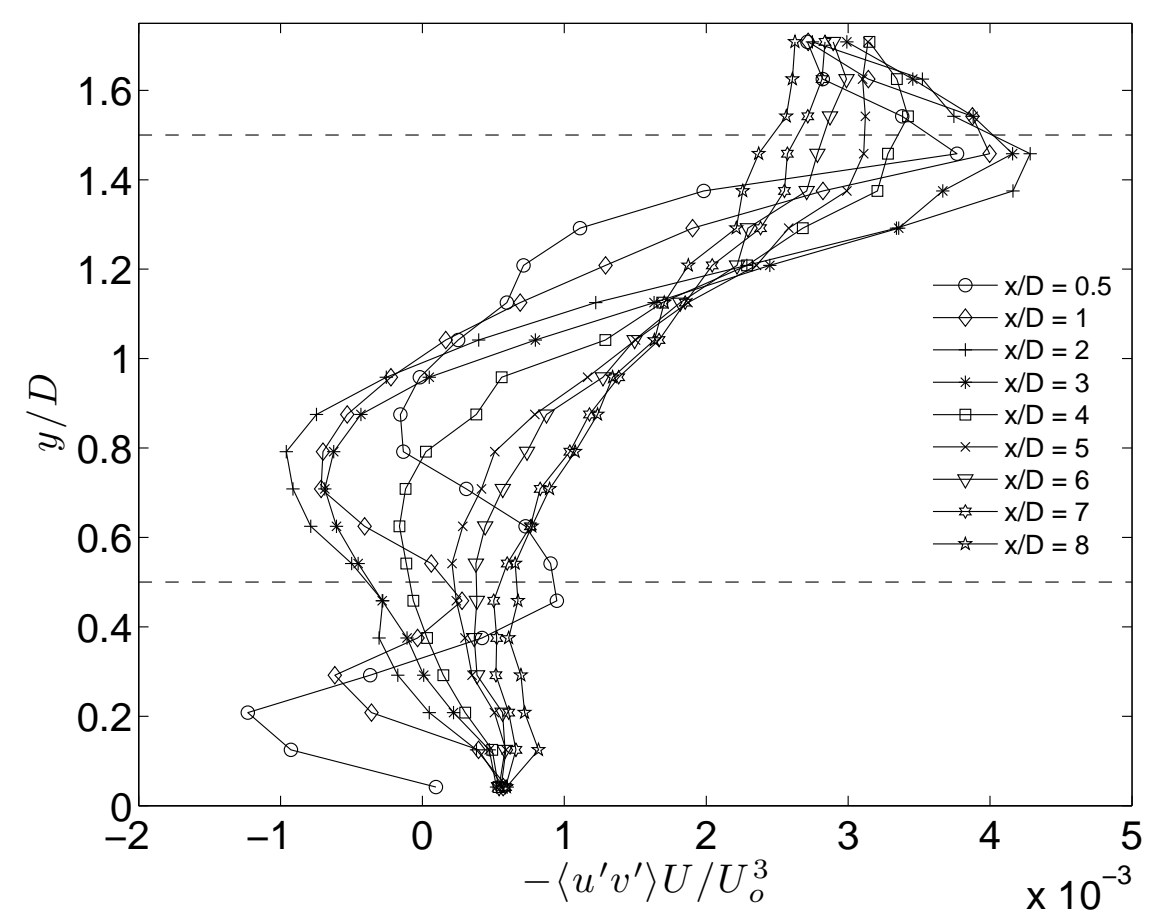

Figure 4.3: Integral form of Kinetic Energy Flux profiles for each measurement location. Data are organized by downstream location. The horizontal dashed lines represent the locations of the top and bottom tip of the rotor blade.

\subsection{Wave Number Spectra}

The process of identifying Markovian behavior in the turbulent flow within a wind turbine array requires examination of the velocity increments. Of the many locations that have been examined, the principles that will be outlined by this process are recognizable at hub height 5 diameters, $x / D=5$, downstream. To visualize the scales of turbulence present at these locations, the normalized energy spectra are plotted in Figure 4.4 for each vertical position at $x / D=5$. Here the one dimensional wave number spectra related to the longitudinal velocity, $F_{11}(k)$, are plotted against wavenumber $k$. In order to obtain $F_{11}(k)$, Taylor's frozen field hypothesis 


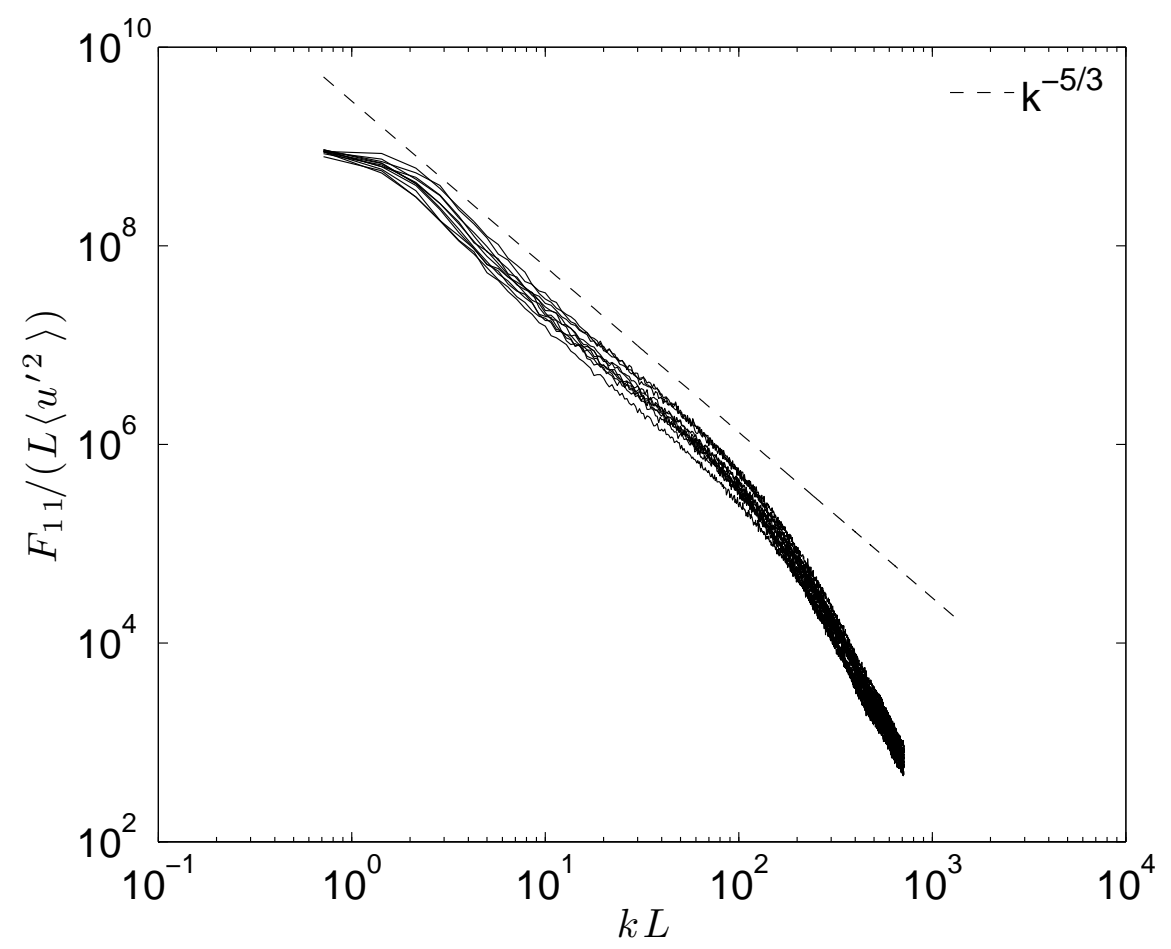

Figure 4.4: Normalized energy spectra for all vertical locations at 5 diameters downstream of the wind turbine.

is applied to the hot-wire data. Here the spectra from every other of the 21 vertical measurement locations for $x / D=5$ are normalized using the integral scale, $L$, which is calculated from the autocorrelation of the fluctuations, and the local second longitudinal central moment, $\left\langle\left(u^{\prime}\right)^{2}\right\rangle . F_{11}(k)$ is then plotted against the normalized wave number. It can be seen that at normalized wave numbers between decades of unity and 100, the spectra in this region follows the $-5 / 3$ dashed line described by Kolmogorov throughout the inertial range, which suggests a separation of turbulent scales. 


\subsection{Comparison of the 2-point and 3-point Conditional Probability}

To verify the existence of a Markovian behavior from equation (2.17), a comparison between the contour plots for $p\left(v_{1}, r_{1} \mid v_{2}, r_{2} ; v_{3}=0, r_{3}\right)$, in blue, and $p\left(v_{1}, r_{1} \mid v_{2}, r_{2}\right)$, in red, is performed. Shown in Figure 4.5, the velocity increment data was taken at $x / D=3$ at hub height and broken into closer scales of $r_{1}=L / 2-\lambda, r_{2}=L / 2$, and $r_{3}=L / 2+\lambda . v_{1}$ and $v_{2}$ are overlaid in the contour plots as well as normalized by $\sigma_{L}$, the standard deviation. The close proximity of the contour lines indicates that equation (2.17) is satisfied and thus the flow is a Markov process for this specific velocity increment scale as Figure 4.5(A) suggests. Furthermore, for scale differences larger than $\lambda$, the flow is Markovian at this location. Horizontal cuts at $v_{2} / \sigma_{L}= \pm 0.2$ are applied to the 3 dimensional pdfs to show the relative proximity of the two. It is clear that the pdfs for both cuts are in perfect agreement, thus again supporting the Markovianity of the flow at this particular location. This can be observed in Figures 4.5(B) and 4.5(C), where $p\left(v_{1}, r_{1} \mid v_{2}, r_{2} ; v_{3}=0, r_{3}\right)$, represented by blue circles, and $p\left(v_{1}, r_{1} \mid v_{2}, r_{2}\right)$, represented by red stars.

In contrast, if the scales of $\Delta r$ are smaller than $\lambda$, then the conditional pdfs no longer satisfy equation (2.17), where $\Delta r=0.3 \lambda$. To be explicit, the velocity increment data is divided into $r_{1}=L / 2-0.3 \lambda, r_{2}=L / 2$, and $r_{3}=L / 2+0.3 \lambda$. As shown in Figure 4.6, the contours for these smaller scale differences show that the conditional pdfs are clearly different. There is a distinct difference between the skewness and kurtosis of the two pdfs, which is highlighted by the horizontal cuts through the pdfs shown in Figure 4.6(B) and 4.6(C). This indicates that the process is no longer Markovian at this smaller scale difference. This type of exploration of the small scale differences is helpful to visualize the scale dependence of the Markov 


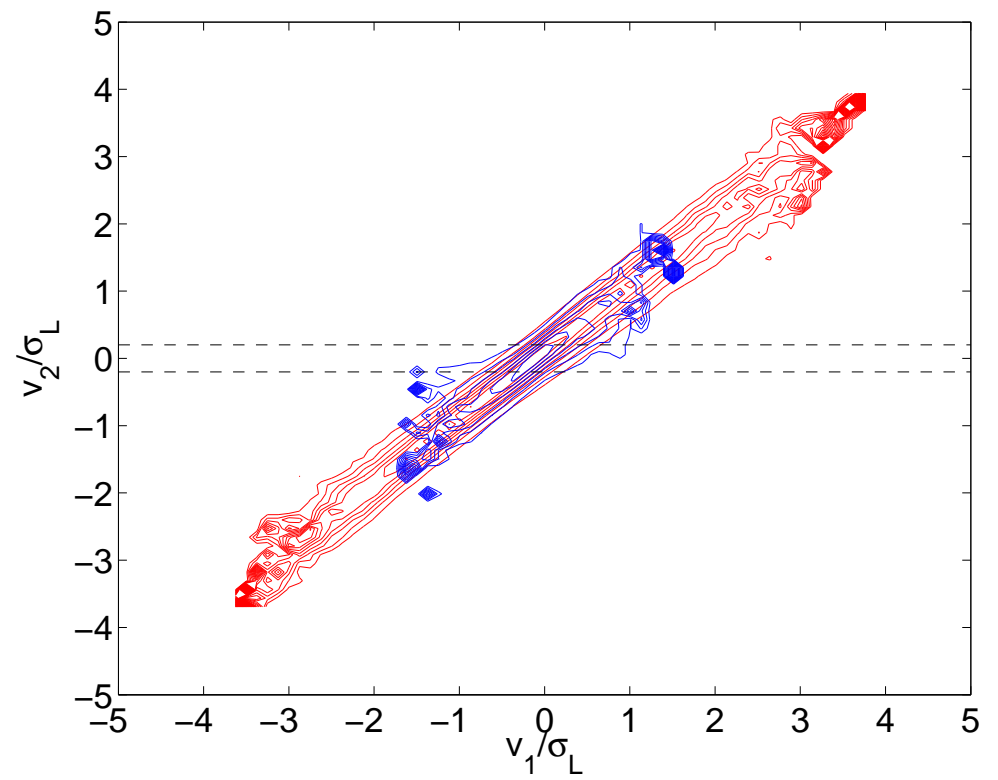

(A) Full conditional pdf comparison
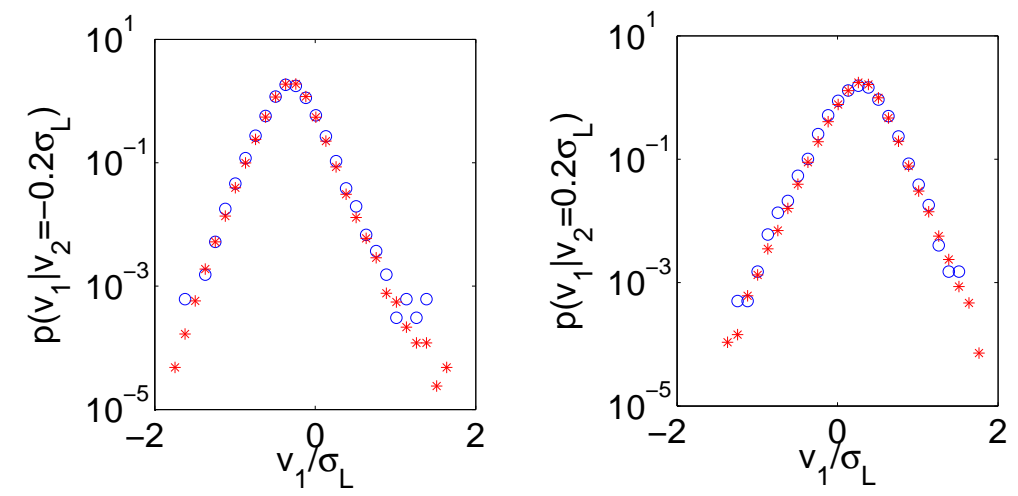

(B) Horizontal cut at $v_{2} / \sigma_{L}=(\mathrm{C})$ Horizontal cut at $v_{2} / \sigma_{L}=$ $-0.2$ 0.2

Figure 4.5: One dimensional conditional pdfs, from $x / D=3$, $p\left(v_{1}, r_{1} \mid v_{2}, r_{2} ; v_{3}=0, r_{3}\right)$ [blue] and $p\left(v_{1}, r_{1} \mid v_{2}, r_{2}\right)$ [red], where $\Delta r=\lambda$ and $v_{3}$ is conditioned to $0 \mathrm{~ms}^{-1}$. 
property but does not give quantitative results. Therefore, to precisely determine the range of scales where Markovian properties hold at each location, a Wilcoxon test is performed.

\subsection{Wilcoxon Test Results}

A Wilcoxon rank-sum test is utilized to explore the range of scales where a Markov process exists. Subsequently, $\left\langle\Delta Q^{*}\right\rangle$ is a function of the physical separations $r_{1}, r_{2}$, and $r_{3}$. It is therefore possible to visualize the dependence of the Markovian behavior on $r_{n}$ and the size of the scale differences, $\Delta r$. In order to get the full picture of the longitudinal velocity increments display of Markovian behavior, $r_{1}$ and $\Delta r$ are varied in the following ranges: $1.5 \lambda \leq r_{1} \leq 20 \lambda$ and $0 \leq \Delta r / \lambda \leq 5$. These variations are shown in Figures 4.7 and 4.8, where variations in the streamwise direction and vertical direction are assessed.

In the following Figures, $\left\langle\Delta Q^{*}\right\rangle$ is plotted as a function of $\Delta r / \lambda$. Figure 4.7 shows the streamwise dependence of $\left\langle\Delta Q^{*}\right\rangle$ at hub height, $y / D=1$. Figures $4.7(\mathrm{~A})$ and $4.7(\mathrm{~B})$ show $\left\langle\Delta Q^{*}\right\rangle$ from the near-wake for $x / D=0.5$ and $x / D=3$ respectively, while Figures $4.7(\mathrm{C})$ and $4.7(\mathrm{D})$ reflect the results from the far-wake at $x / D=5$ and $x / D=8$. A cartoon has been included in each subfigure to indicate the particular measurement location. In the location nearest to the hub at $x / D=0.5$, the values of $\left\langle\Delta Q^{*}\right\rangle$ converge to 1 at $\Delta r=5 \lambda$ for all scales $r_{1}$, as shown in Figure 4.7(A). At this location it is seen that for scales below $\Delta r=2 \lambda$ the values for $\left\langle\Delta Q^{*}\right\rangle$ increase rapidly and the Markov behavior falls apart for these smaller scales of turbulence. This departure appears to be independent of $r_{1}$ and contain the same distribution as these all collapse to the same curve. The smallest initial scales, $r_{1}$, take the 


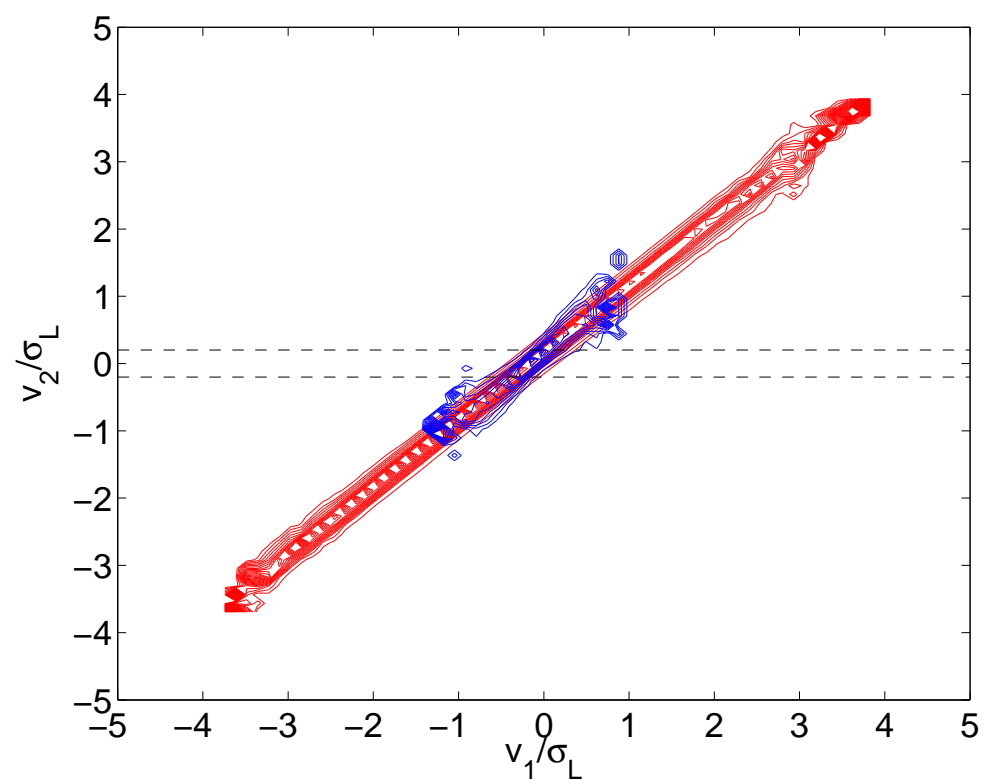

(A) Full conditional pdf comparison
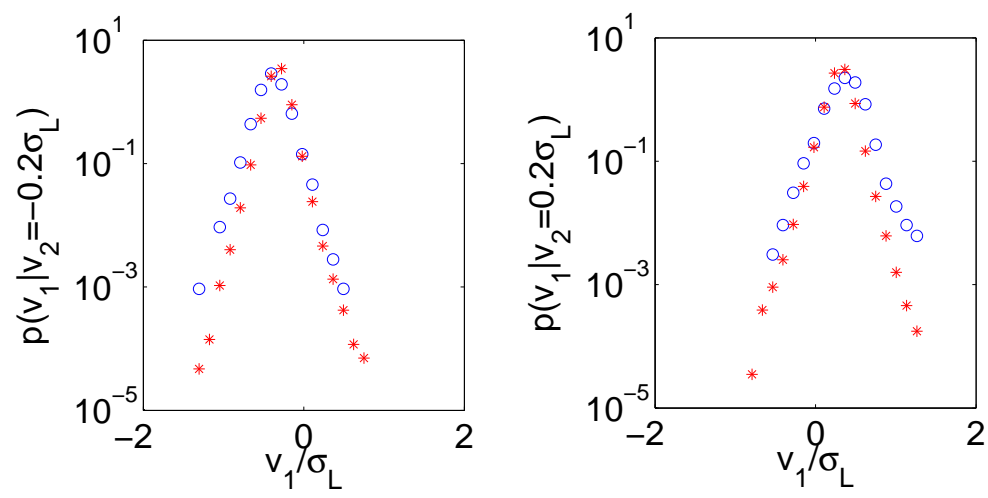

(B) Horizontal cut at $v_{2} / \sigma_{L}=(\mathrm{C})$ Horizontal cut at $v_{2} / \sigma_{L}=0.2$ $-0.2$

Figure 4.6: One dimensional conditional pdfs, from $x / D=3$, $p\left(v_{1}, r_{1} \mid v_{2}, r_{2} ; v_{3}=0, r_{3}\right)$ [blue], and $p\left(v_{1}, r_{1} \mid v_{2}, r_{2}\right)$ [red], where $\Delta r=0.3 \lambda$ and $v_{3}$ is conditioned to $0 \mathrm{~ms}^{-1}$. 


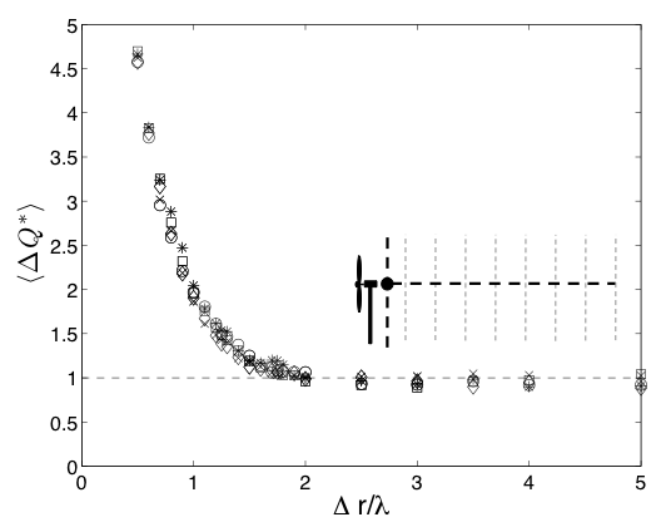

(A) $x / D=0.5$

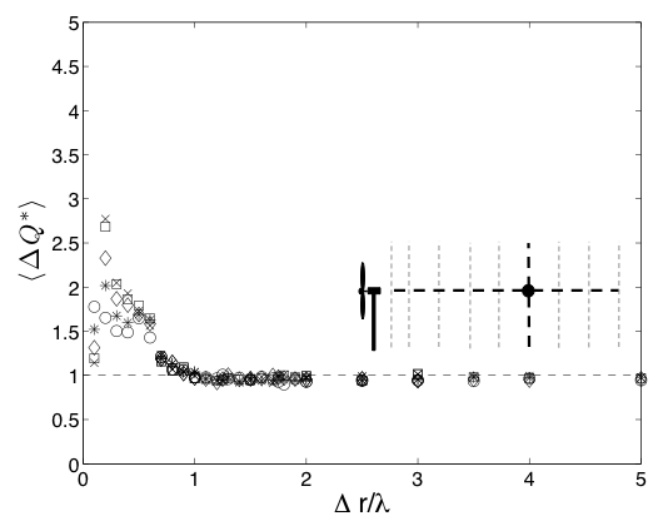

(C) $x / D=5$

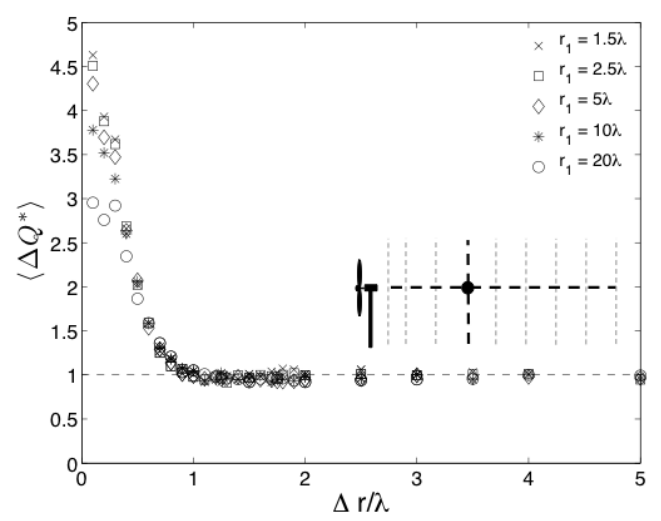

(B) $x / D=3$

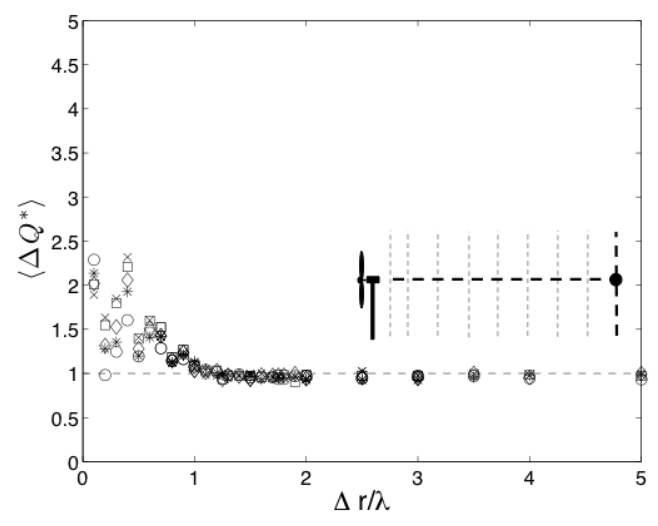

(D) $x / D=8$

Figure 4.7: Wilcoxon test results showing downstream trend at hub height, $y / D=1$.

longest to reach 1 showing that near to the hub the flow is characterized by large scale intermittency. The level of departure from the small scale Markovian behavior may be an indication of why Barthelmie et al. found that despite differences in the measurement height and period, overall agreement is better between the turbulence intensity derived from power measurements and the meteorological mast than with those derived from data from the nacelle anemometers [16].

Further downstream in Figure $4.7(\mathrm{~B})$ at $x / D=3$, the Wilcoxon test results 
show that all values of $r_{1},\left\langle\Delta Q^{*}\right\rangle=1$ for scale differences of $\Delta r>1.1 \lambda$. At this location the rapid departure of $\left\langle\Delta Q^{*}\right\rangle$ from one is seen again, although this time the departure begins at scale differences of $\Delta r>1.1 \lambda$. A similar result is seen in Figure $4.7(\mathrm{C})$, where the value of $\left\langle\Delta Q^{*}\right\rangle=1$ for scale differences of $\Delta r>\lambda$. This behavior is taken as an indication that the wake has begun to recover and that the large energy entrainment, present near the hub has decreased significantly. This result means that the conditional probability density functions are equivalent for these scales and thus over this range of scales the flow is a Markov process.

In Figure 4.7(B) and Figure 4.7(C) it is shown that for scales greater than, $\Delta r \geq \lambda$, the wake is a Markov process. It should be noted that once a $\left\langle\Delta Q^{*}\right\rangle$ value of one is reached, the Markov properties are not dependent upon the selection of $r_{1}$. This is a similar result to what has been shown to be the case for homogenous, isotropic turbulence in prior investigations [22, 24].

For $x / D=8$, the values of $\Delta r$ where $\left\langle\Delta Q^{*}\right\rangle=1$ increase to $1.3 \lambda$ as shown in Figure 4.7(D). At this location the wake of the turbine has remediated. These regions are characterized by low levels of shear. It is likely that at this point the level of shearing occurring is too low and there is a significant level of mixing between the ABL and the wake of the turbine.

As the flow moves downstream of the turbine, in Figures 4.7(B), 4.7(C), 4.7(D), the value of $\left\langle\Delta Q^{*}\right\rangle$ for scale differences $\Delta r / \lambda<1$ approach unity. This behavior also demonstrates dependence upon the selection of the initial scale, $r_{1}$, where results from larger values of $r_{1}$ are closer to $\left\langle\Delta Q^{*}\right\rangle=1$ relative to the results from smaller values of $r_{1}$, most clearly seen in Figure 4.7(D). This can be related to the lower values of turbulent kinetic energy production in Figure 4.2 and kinetic energy flux 
from Figure 4.3 found at these locations. As production of kinetic energy decreases, the entrainment of large scales diminishes leading to a more organized cascade where the scale differences below the Taylor micro-scale begin to demonstrate Markovian behavior. The reduced kinetic energy flux has a similar effect on the turbulence statistics, where the influence of non-local energy entrainment is reduced allowing for well developed turbulent cascade at smaller scale differences.

Figure 4.8 shows the Wilcoxon test results at $x / D=0.5$ (left) and 5(right) for the top-tip and bottom-tip, using the two corresponding locations at hub height for reference. Figures $4.8(\mathrm{~A})$ and $4.8(\mathrm{~B})$ show the Wilcoxon test results at the top-tip, $4.8(\mathrm{C})$ and $4.8(\mathrm{D})$ show the results at hub height, and $4.8(\mathrm{E})$ and $4.8(\mathrm{~F})$ show the results at the bottom-tip.

At the top-tip height and $x / D=0.5$ downstream from the rotor, Figure 4.8(A), the results from the Wilcoxon test show a convergence of $\left\langle\Delta Q^{*}\right\rangle$ to 1 at $\Delta r=\lambda$. There is evidence of scale dependence as the values of $\left\langle\Delta Q^{*}\right\rangle$ begin to diverge from 1 once the values of $\Delta r$ become greater than $2.5 \lambda$. The first values to diverge are the smallest values of $r_{1}=1.5 \lambda$ and $2.5 \lambda$. This departure is hypothesized to be caused by the presence of tip vortices. These structures arrive at regular intervals in the small range for which these scale show Markovian properties, but as the value of $\Delta r$ becomes larger than the scale of the tip vortices, they begin to appear irregularly in the pdfs and thus cause a divergence of $\left\langle\Delta Q^{*}\right\rangle$. For the larger values of $r_{1}$ these structures exert an evenly distributed influence on the pdfs and thus do not cause a divergence of $\left\langle\Delta Q^{*}\right\rangle$.

For the bottom tip locations nearest to the rotor, shown in Figure 4.8(E), the values of $\left\langle\Delta Q^{*}\right\rangle$ converge to 1 at $\Delta r=1.4 \lambda$, revealing a Markov process for these 


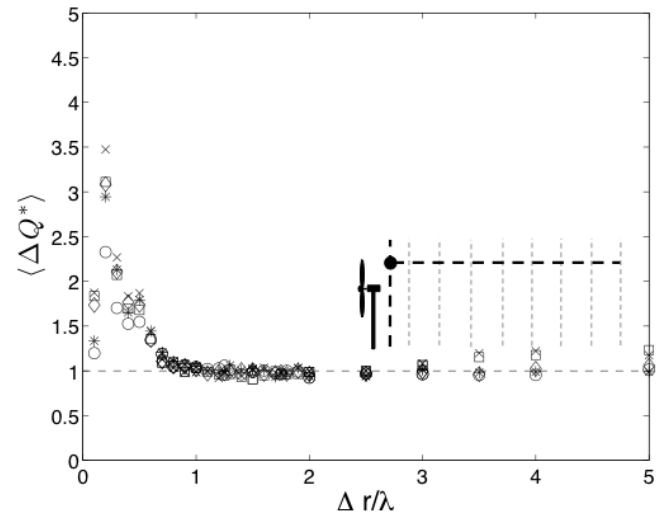

(A) $x / D=0.5$ and $y / D=1.5$

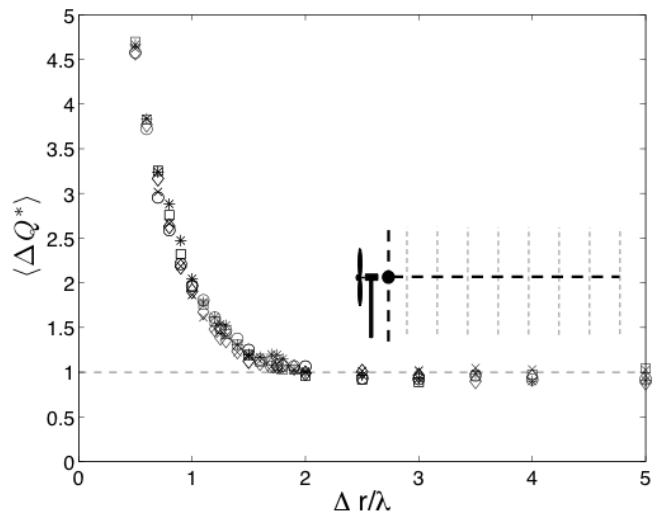

(C) $x / D=0.5$ and $y / D=1$

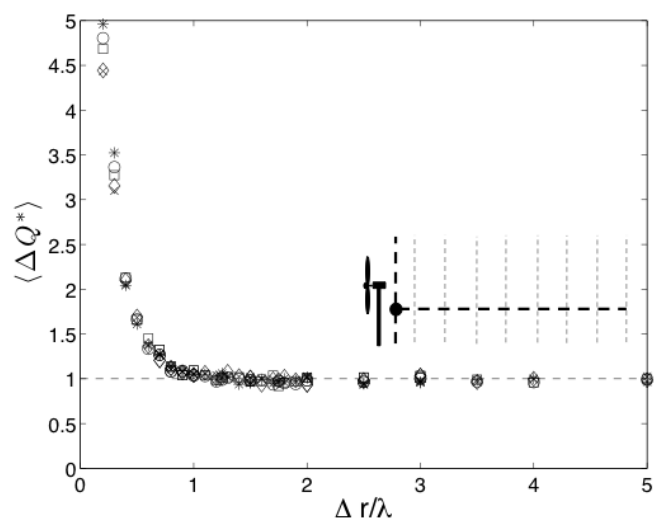

(E) $x / D=0.5$ and $y / D=0.5$

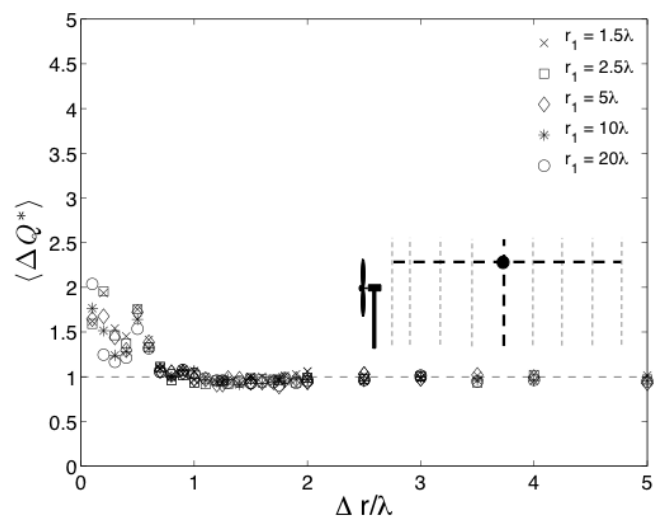

(B) $x / D=5$ and $y / D=1.5$

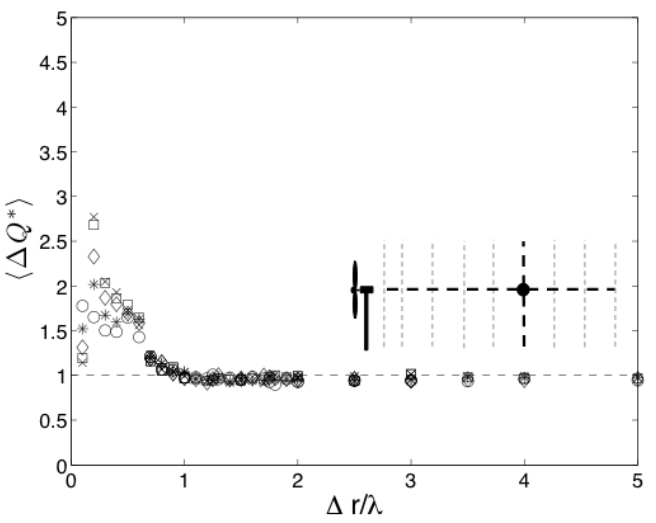

(D) $x / D=5$ and $y / D=1$

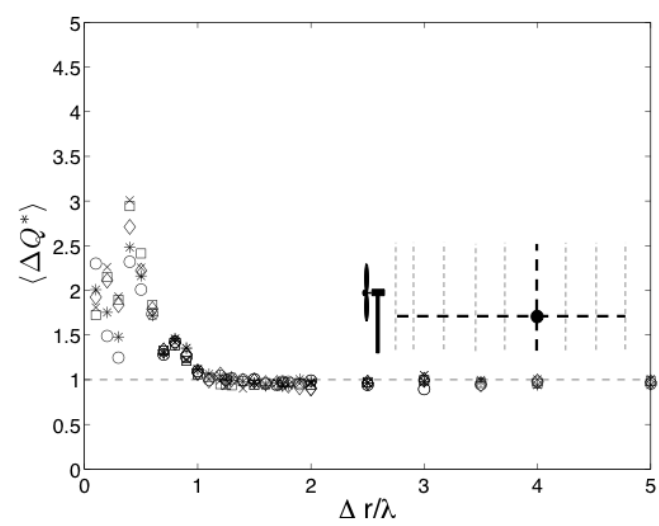

(F) $x / D=5$ and $y / D=0.5$

Figure 4.8: Wilcoxon test results showing vertical location at top-tip, hub height and bottom-tip for $x / D=0.5$ (a, c, e) and $x / D=5(\mathrm{~b}, \mathrm{~d}, \mathrm{f})$. 
scales at this location. In Figure 4.8(E) the influence of tip vortices are not apparent due to the presence of the turbine mast. Yet, the trend of $\left\langle\Delta Q^{*}\right\rangle$ at lower tip reveals heterogenous turbulence directly behind the turbine that is likely caused by the irregular interaction of tip vortices from the blades with the mast, as indicated by the departure of $\left\langle\Delta Q^{*}\right\rangle$ from 1 at the Taylor micro-scale.

Once the flow reaches $x / D=5$ in Figure 4.8(B) the tip vortices have dissipated and the values of $\left\langle\Delta Q^{*}\right\rangle=1$ at $\Delta r=\lambda$ for all scales of $r_{1}$. Here a slight difference between the two plots can be seen in the behaviors of the smaller scale differences. For the location further away from the rotor, $\left\langle\Delta Q^{*}\right\rangle$ values are relatively closer to 1 for the smaller scale differences $\Delta r$. This result is taken as an indication that the larger scale structures present near to the rotor have begun to breakdown due to the inherent instability of the turbulence cascade. This behavior is observed in Figures 4.8(D) and 4.8(F) as well.

As illustrated by Figures 4.7 and 4.8 the presence of a Markov process is highly dependent upon the streamwise and wall normal position. To illustrate this dependence a contour plot of all of the Wilcoxon test results within the wake of the turbine is shown in Figure 4.9. Here, the smallest values of $\Delta r$ are given for the point at which $\Delta Q^{*}=1$. From this figure, it can be seen that the largest values of $\left\langle\Delta Q^{*}\right\rangle$ are located directly behind the hub. The near-wake region is characterized by non-Markovian behavior at the smallest scales and extends 2-3 diameters downstream.

With Figure 4.9 it is possible to track the flow behavior at the top and bottom tips as it develops downstream of the wind turbine array in terms of its Markovianity. In this figure, dashed lines are used to identify the top-tip, hub height, and bottom 


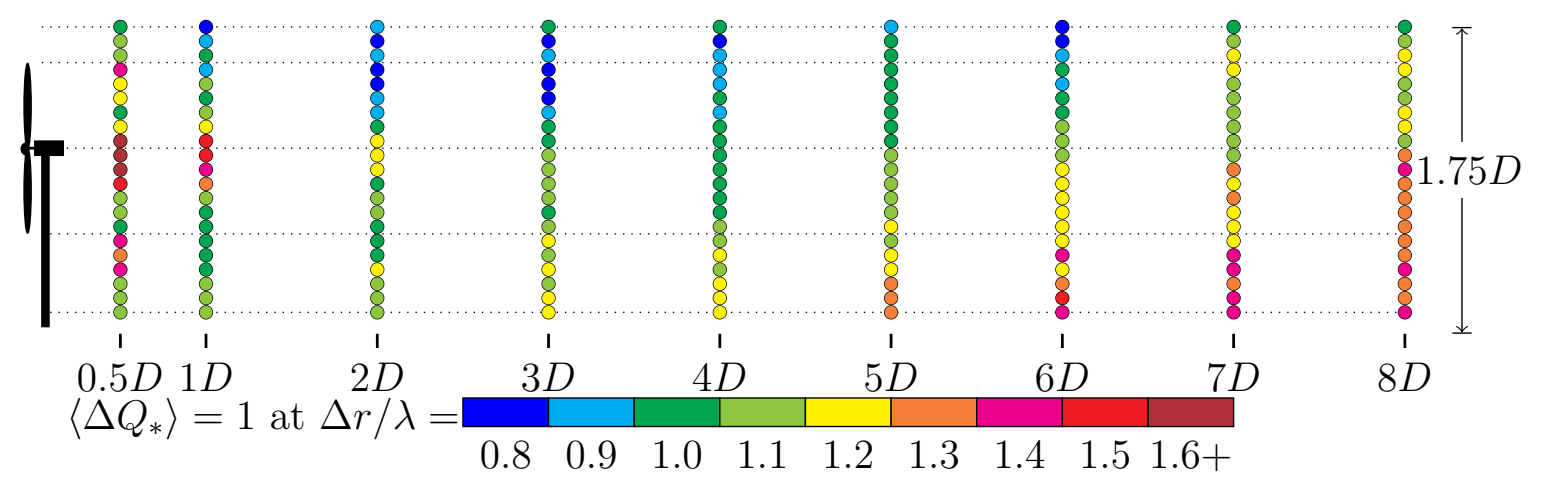

Figure 4.9: Spatial representation of Wilcoxon test results.

tip locations. It can be seen that the condition for which $\Delta Q^{*}$ is equal to unity is satisfied for several locations at values of $\Delta r / \lambda$ approximately equal to one. One diameter downstream the level of shear within the boundary layer has overcome the strength of the tip vortices leading a convergence of $\left\langle\Delta Q^{*}\right\rangle$ to 1 at scale differences of $\Delta r=\lambda$ for both the top and bottom tips. The flow continues to exist as a Markov process for all locations between $x / D=2$ and $x / D=5$. At $x / D=5$ the values of $\left\langle\Delta Q^{*}\right\rangle$ begin to diverge from 1 near the wall. The number of locations that show this divergence increases in wall-normal direction with each downstream location. Until signs of divergence occur at hub height for $x / D=8$. For measurement locations below the bottom tip, this is taken as an indication of increased levels on non-local interaction as the lower or surface boundary layer begins to mix with the wake of the wind turbine. Locations above the top tip of the rotor beginning at $y / D>$ 1.5 downstream, results show no deviation from $\left\langle\Delta Q^{*}\right\rangle=1$ with the exception of $x / D=8$, where $\left\langle\Delta Q^{*}\right\rangle=1$ at scale differences on the order of $1.5 \lambda$.

The markovianity of the near wake is explored by analyzing points located vertically at $x / D=0.5$. Starting with locations just above the floor, $\left\langle\Delta Q^{*}\right\rangle=1$ at scales above the Taylor micro-scale. Within the lower (wall) boundary layer, these values 
vary from $\Delta r / \lambda=1.1$ to 1.4 past the lower tip until just below hub height, where $\left\langle\Delta Q^{*}\right\rangle$ increases to values of $\Delta r / \lambda>2$. This trend continues behind and just past the hub. The slight asymmetry between locations above and below the hub show that the turbine mast and the wall play a role in the flow characteristics. Where below the hub values of $\left\langle\Delta Q^{*}\right\rangle$ rarely converge to 1 at $\Delta r / \lambda=1$, while above hub height there are several locations where flow is Markovian for values of $\Delta r / \lambda<1$. Where starting at $x / d=2\left\langle\Delta Q^{*}\right\rangle$ values reach as low as $0.8 \lambda$.

Perhaps the most interesting results are found at $x / D=4$ where the flow is showing Markovian properties down to the Taylor micro-scale at all locations above the bottom tip of the rotor. This location is often marked as the end of the nearwake and beginning of the far-wake region. It shows complete scale independence of the Markov property, as all scales of $r_{1}$ and subsequent $\Delta r$ are Markovian at $\lambda$. It is also interesting to note that this location falls within the standard spacing of wind turbines, offering the possibility of usable information for more accurate power prediction and maintenance scheduling at these locations.

\subsection{Local Reynolds number \& energy spectra}

The local Reynolds number for each measurement location has been calculated using local mean velocity and the local Taylor micro-scale,

$$
R e_{\lambda}=\frac{U \lambda}{\nu}
$$

$R e_{\lambda}$ as shown in Figure 4.10. For locations within the near-wake region, $R e_{\lambda}$ ranges from $108-1377$. The smallest values being found at hub height and largest values 


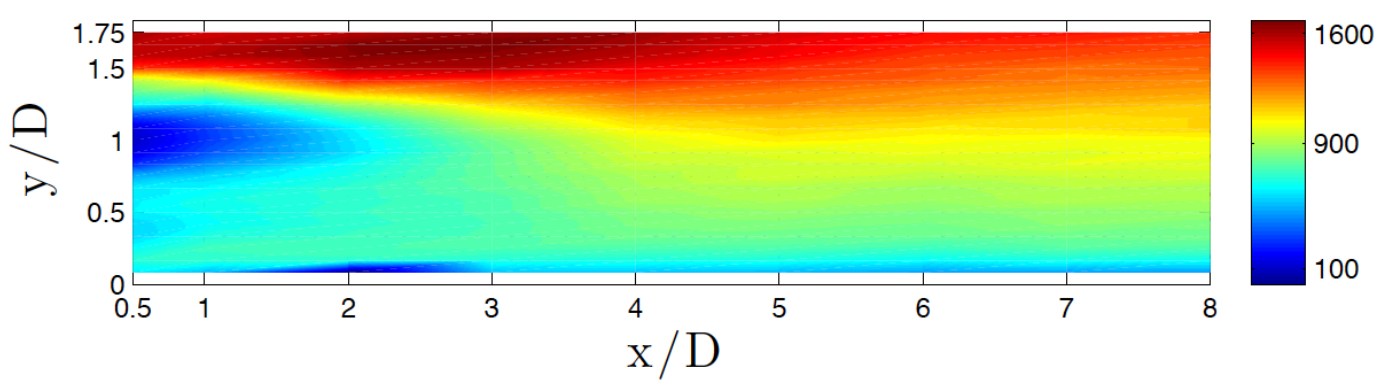

Figure 4.10: Local Reynolds number based on the Taylor micro-scale, $R e_{\lambda}$, for each measurement location.

above the top tip. Moving downstream the local Reynolds number increases at all locations with the exception locations near the wall where the values remain relatively unchanged. $R e_{\lambda}$ values reach a maximum value of $R e_{\lambda}=1683$ at $x / D=4$ and $y / D=1.5$, which corresponds to the locations that show the largest range of scales which demonstrate Markov behavior. As can be seen from the results of the Wilcoxon test, downstream locations that yield the most consistent Markovian properties occur at $x / D=4$. For locations that show Markov process behavior down to the local Taylor micro-scale, the minimum value of $R e_{\lambda}$ is found to be, $R e_{\lambda} \approx 550$ or greater.

As shown in Figure 4.10, the locations nearest to the rotor, $x / D=0.5$, the local Reynolds numbers are consistently lower than the $R e_{\lambda}=550$ mark, dropping to the lowest value of $R e_{\lambda}=108$ at hub height. These lower values of the Reynolds number are drawn from the smaller values of the Taylor micro-scale for these locations, which indicates the influence of dissipation in these locations. Lower Reynolds number and smaller Taylor micro-scale are related to the lower values of longitudinal velocities found in these locations.

At $x / D=4$, most locations within the vertical profile show Markovian behavior 


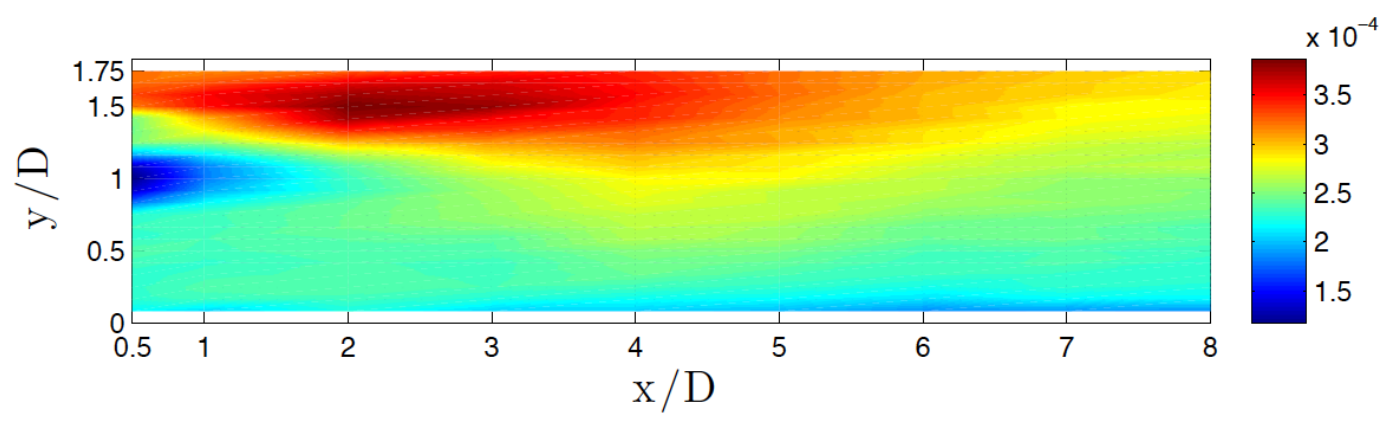

Figure 4.11: Local Taylor micro-scale, $\lambda$, for each measurement location

down to the Taylor micro-scale. This occurs at locations across the rotor diameter and above, an important implication for potential power prediction. For these locations the Taylor micro-scale is $\lambda \approx 2.5 \times 10^{-3} \mathrm{~m}$ or greater as shown Figure 4.11; the local $R e_{\lambda}$ is above 850 .

\subsection{Flattened Wave Number Spectra}

To further investigate the flow characteristics that affect the Markovian properties of the wake, a second look at the normalized energy spectra for specific locations of interest is taken. Previously in Figure 4.4, the spectra were presented in a traditional manner, but in order to identify the true length of the inertial sub-range, the spectra are multiplied by $k^{5 / 3}$, where $k$ is the wave number . The effect of this manipulation is that for the range where the spectra have a $-5 / 3$ slope, the modified spectra will lay flat. From the results of the Wilcoxon test, it is found that the turbulence cascade $0.5 D$ downstream of the hub is a Markov process for scale differences of $\Delta r=5 \lambda$. This value is reflected in Figure 4.12 where the inertial range is very short. This result is quite different from the Wilcoxon test result $2 \mathrm{D}$ downstream at the top tip, where $\left\langle\Delta Q^{*}\right\rangle=1$ at $\Delta r=0.8 \lambda$. The energy spectra from this location 


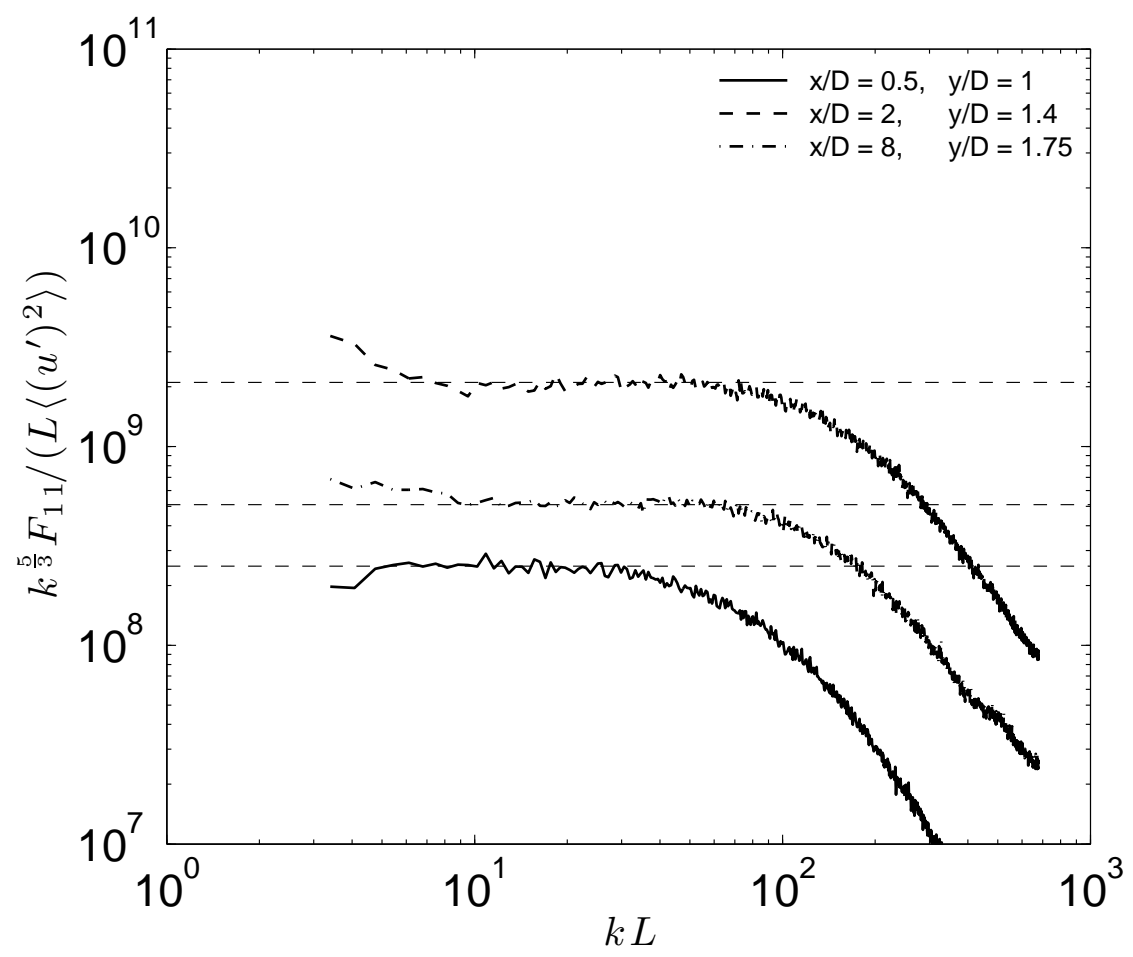

Figure 4.12: Flattened energy spectra have been shifted vertically to highlight the inertial sub-range at critical locations in the wake $x / D=0.5$ at $y / D=1$, $x / D=2$ at $y / D=1.4, x / D=8$ at $y / D=1.75$

shows a longer inertial sub-range of nearly a decade. This range is nearly the same length as the inertial range found in the near free stream turbulence at $x / D=8$ above the top tip. This figure leads to the conclusion that, for a larger inertial sub-range the process will be Markovian for smaller scale differences, $\Delta r$. 


\section{Chapter 5}

\section{Conclusions}

Hot-wire anemometry data obtained in a wind tunnel experiment was used to evaluate the behavior of the turbulence statistics behind the center turbine in the exit row of a $3 \times 3$ wind turbine array. Profiles of mean velocity, turbulent kinetic energy production, and kinetic energy flux are used to characterize the wake. By comparing the conditional pdfs for $p(v 1, r 1 \mid v 2, r 2 ; v 3=0, r 3)$ and $p(v 1, r 1 \mid v 2, r 2)$, the wake is shown to demonstrate the presence of a Markov process for scale differences of $\Delta r \sim \lambda$

A Wilcoxon rank-sum test is preformed for numerous scale differences to investigate the equality of 2- and 3-point conditional pdfs for each measurement location. Results show that for hub height locations at $x / D=0.5$ and $x / D=1$, the flow is not a Markov process. Locations in the near-wake demonstrate incremental scale, $r$, dependence for the Markov process which is attributed to the geometric influence of the wind turbine and periodic structures caused by the rotation of the blades such as tip vortices. For other locations downstream of turbine array, the Wilcoxon test results show that the wind turbine wake is a Markov process for scales down to and occasionally below the Taylor microscale, $\lambda$. There is an apparent asymmetry between locations above and below hub height. Below hub height, $\left\langle\Delta Q^{*}\right\rangle$ equals 
unity at $\Delta r=1.2 \lambda$. For scales larger than this, the turbulent cascade is a Markov process at all measurement locations. In contrast, above hub height, $\left\langle\Delta Q^{*}\right\rangle=1$ at $\Delta r=1$ or less.

Measurements show that the presence of energy entrainment regions in the flow has a negative impact on the emergence of Markovian behavior at the scale differences smaller than $\lambda$, but does not eliminate the process altogether. For the locations that show Markov process behavior down to the local Taylor microscale, the minimum value of $R e_{\lambda}$ is found to be, $R e_{\lambda} \approx 550$ or greater. At $x / D=4$, most locations within the vertical profile that show Markovian behavior down to the Taylor microscale. This occurs at locations across the rotor diameter and above, an important implication for potential power prediction as the properties of the flow at these locations will serve as the inlet conditions for the next wind turbine. It is also shown that there is a correlation between the presence of an inertial sub-range and the Markovian process. Where a longer inertial sub-range leads to an extension of the range of the Markov process into smaller scales of turbulence.

As it is applied to wind energy, the Markovian nature of high reynolds number turbulence holds great potential for improving maintenance schedules as well as improving the overall efficiency of the turbines. It has been shown in previous work [11-13] that prediction of power production becomes difficult with current techniques, where point measurements are taken upstream of a large scale wind farm. Once the wind enters the array, the complex interaction of the subsequent wind turbine wakes lead to poor estimation of power output. In this study, the downstream vertical locations that show the most consistent Markov properties are found to occur at four diameters downstream of a wind turbine within an array. 
With the experimental spacing of the turbines of $x / D=7$ the resulting statistics are taken to represent the flow behavior found in an "infinite turbine array" [28]. In combination with the fact that downstream spacing of turbines in a large wind farm is often larger than 4 diameters, offers a potential solution to the issues experienced by large scale arrays. 


\section{Chapter 6}

\section{Future Work}

Now that the extent of the Markovian nature of the wake has been identified, it is possible to perform an analysis of the Conditional Moments at those scales. The Chapman-Kolmogorov equation which describes the r-dependence of each conditional moment can be expressed in differential form, as follows:

$$
-r \frac{\partial}{\partial r} p\left(v, r \mid v_{0}, r_{0}\right)=\sum_{k=1}^{\infty}\left(-\frac{\partial}{\partial v}\right) D_{k}(v, r) p\left(v, r \mid v_{0}, r_{0}\right)
$$

This form of the Chapman-Kolmogorov equation is called the Kramers-Moyal expansion. For more information on how this equation is derived, the reader is referred

to [36]. Through the use of this equation, it is possible to evaluate the conditional moments, $M_{k}(v, r, \Delta r)$.

\subsubsection{Fokker-Planck Equation}

To solve the Kramers-Moyal expansion is nearly impossible without knowing all of the Kramers-Moyal coefficients, $D_{k}$. If evaluated at the limit as $r$ approaches zero, the value of $D_{2}>>D_{4}$ then, according to the Pawula theorem, the Kramers-Moyal can be reduced to the first two terms of the equation yielding the Fokker-Planck 
equation [36]. The resulting Fokker-Planck Equation:

$$
-r \frac{\partial}{\partial r} p\left(v, r \mid v_{0}, r_{0}\right)=-\frac{\partial}{\partial v} D_{1}(v, r) p\left(v, r \mid v_{0}, r_{0}\right)+\frac{\partial^{2}}{\partial v^{2}} D_{2}(v, r) p\left(v, r \mid v_{0}, r_{0}\right)
$$

where $D_{1}$ is the drift term and $D_{2}$ is the diffusion term of the expansion of conditional PDF, $p\left(v, r \mid v_{0}, r_{0}\right)$. Due to the Markov process discovered in the wake, the solution to this equation is considered to be a governing equation for the downstream evolution of the probability density function. 


\section{Bibliography}

[1] WWEA, "The world wind energy report 2012," The world wind energy association, 2013.

[2] R. Saidur, M. Islam, N. Rahim, and K. Solangi, "A review on global wind energy policy," Renewable and Sustainable Energy Reviews, vol. 14, no. 7, pp. 1744 1762, 2010.

[3] I. Chapman, "The end of peak oil? why this topic is still relevant despite recent denials," Energy Policy.

[4] H. Waisman, J. Rozenberg, O. Sassi, and J.-C. Hourcade, "Peak oil profiles through the lens of a general equilibrium assessment," Energy Policy, vol. 48, no. 0, pp. $744-753,2012$.

[5] M. Islam, S. Mekhilef, and R. Saidur, "Progress and recent trends of wind energy technology," Renewable and Sustainable Energy Reviews, vol. 21, no. 0, pp. $456-468,2013$.

[6] T. Burton, N. Jenkins, D. Sharpe, and E. Bossanyi, Wind energy handbook. John Wiley \& Sons, 2011.

[7] R. J. Barthelmie and S. C. Pryor, "Challenges in predicting power output from offshore wind farms.," Journal of Energy Engineering, vol. 132, no. 3, pp. 91 $103,2006$.

[8] E. Anahua, S. Barth, and J. Peinke, "Markovian power curves for wind turbines," Wind Energy, vol. 11, no. 3, pp. 219-232, 2008.

[9] S. R. Arwade, M. A. Lackner, and M. D. Grigoriu, "Probabilistic models for wind turbine and wind farm performance," Journal of Solar Energy Engineering, vol. 133, no. 4, p. 041006, 2011.

[10] E. Byon and Y. Ding, "Season-dependent condition-based maintenance for a wind turbine using a partially observed markov decision process," Power Systems, IEEE Transactions on, vol. 25, pp. 1823 -1834, nov. 2010. 
[11] Y. Ma, T. Runolfsson, and J. Jiang, "Cluster analysis of wind turbines of large wind farm with diffusion distance method," Renewable Power Generation, IET, vol. 5, pp. 109 -116, March 2011.

[12] R. Barthelmie, L. Folkerts, F. Ormel, P. Sanderhoff, P. Eecen, O. Stobbe, and N. Nielsen, "Offshore wind turbine wakes measured by sodar," J. Atmos. Ocean. Technol. (USA), vol. 20, no. 4, pp. 466 - 77, 2003.

[13] A. Crespo, J. Hernandez, and S. Frandsen, "Survey of modelling methods for wind turbine wakes and wind farms," Wind Energy, vol. 2, no. 1, pp. 1-24, 1999 .

[14] S. Frandsen, R. Barthelmie, S. Pryor, O. Rathmann, S. Larsen, J. Hojstrup, and M. Thogersen, "Analytical modelling of wind speed deficit in large offshore wind farms," Wind Energy (UK), vol. 9, no. 1-2, pp. 39 - 53, 2006/01/.

[15] L. Vermeer, J. Sorensen, and A. Crespo, "Wind turbine wake aerodynamics," Progress in Aerospace Sciences, vol. 39, no. 6-7, pp. 467 - 510, 2003.

[16] R. J. Barthelmie, S. T. Frandsen, M. N. Nielsen, S. C. Pryor, P.-E. Rethore, and H. E. Jørgensen, "Modelling and measurements of power losses and turbulence intensity in wind turbine wakes at middelgrunden offshore wind farm," Wind Energy, vol. 10, no. 6, pp. 517-528, 2007.

[17] A. Nawroth and J. Peinke, "Multiscale reconstruction of time series," Physics Letters A, vol. 360, no. 2, pp. 234 - 237, 2006.

[18] F. Ghasemi, M. Sahimi, J. Peinke, and M. Reza Rahimi Tabar, "Analysis of non-stationary data for heart-rate fluctuations in terms of drift and diffusion coefficients," J. Biol. Phys. (USA), vol. 32, no. 2, pp. 1117 - 28, 2006.

[19] T.-T. Leung, "A markov-modulated stochastic control problem with optimal multiple stopping with application to finance," (Piscataway, NJ, USA), pp. 559 - 66, 2010.

[20] W. Fleming, "Controlled markov processes and mathematical finance," (Dordrecht, Netherlands), pp. 407 - 46, 1999.

[21] J. McCauley, K. Bassler, and G. Gunaratne, "Markov processes, hurst exponents, and nonlinear diffusion equations: With application to finance," Physica A (Netherlands), vol. 369, no. 2, pp. $343-53,2006$. 
[22] C. Renner, J. Peinke, and R. Friedrich, "Experimental indications for markov properties of small-scale turbulence," J. Fluid Mech. (UK), vol. 433, pp. 383 409, 2001.

[23] M. Siefert and J. Peinke, "Joint multi-scale statistics of longitudinal and transversal increments in small-scale wake turbulence," Journal of Turbulence, p. N50, 2006.

[24] M. Tutkun and L. Mydlarski, "Markovian properties of passive scalar increments in grid-generated turbulence," New Journal of Physics, vol. 6, no. 1, p. 49, 2004.

[25] W. Zhang, C. Markfort, and F. Porté-Agel, "Near-wake flow structure downwind of a wind turbine in a turbulent boundary layer," Experiments in Fluids, vol. 52, pp. 1219-1235, 2012.

[26] L. P. Chamorro and F. Porte-Agel, "A wind-tunnel investigation of windturbine wakes: Boundary-layer turbulence effects," Boundary-Layer Meteorology, vol. 132, no. 1, pp. 129 - 149, 2009.

[27] R. B. Cal, J. Lebrón, L. Castillo, K. Hyung Suk, and C. Meneveau, "Experimental study of the horizontally averaged flow structure in a model wind-turbine array boundary layer.," Journal of Renewable and Sustainable Energy, vol. 2, no. 1, p. 013106, 2010.

[28] L. P. Chamorro and F. Porté-Agel, "Turbulent flow inside and above a wind farm: A wind-tunnel study," Energies, vol. 4, no. 11, pp. 1916-1936, 2011.

[29] A. N. Kolmogorov, "Dissipation of energy in locally isotropic turbulence," Doklady Akad. Nauk SSSR, vol. 32, no. 16, 1941.

[30] W. K. George, "Lectures in turbulence for the 21st century," 2010.

[31] G. I. Taylor, "The spectrum of turbulence," Proceedings of the Royal Society of London. Series A - Mathematical and Physical Sciences, vol. 164, no. 919, pp. 476-490, 1938.

[32] L. Mydlarski and Z. Warhaft, "On the onset of high-reynolds-number gridgenerated wind tunnel turbulence," Journal of Fluid Mechanics, vol. 320, pp. $331-368,1996$.

[33] G. I. Taylor, "Statistical theory of turbulence," Proceedings of the Royal Society of London. Series A - Mathematical and Physical Sciences, vol. 151, no. 873, pp. 421-444, 1935 . 
[34] H. Makita, "Realization of a large-scale turbulence field in a small wind tunnel," Fluid Dynamics Research, vol. 8, no. 1-4, pp. 53 - 64, 1991.

[35] C. M. N. Hamilton, H. Suk Kang and R. Cal, "Statistical analysis of kinetic energy entrainment in a model wind turbine array boundary layer," Journal of Renewable and Sustainable Energy, vol. 4, no. 6, p. 063105, 2012.

[36] H. Risken, The Fokker-Planck Equation: Methods of Solutions and Applications. Springer Series in Synergetics, Springer, 2nd ed. 1989. 3rd printing ed., Sept. 1996. 